Check for updates

Cite this: Phys. Chem. Chem. Phys., 2020, 22, 12044

Received 22nd January 2020, Accepted 1st May 2020

DOI: $10.1039 / \mathrm{d} 0 \mathrm{cp00376j}$

rsc.li/pccp

\title{
On the polarization of ligands by proteins $\uparrow$
}

\author{
Soohaeng Yoo Willow, ${ }^{a}$ Bing Xie, ${ }^{a}$ Jason Lawrence, ${ }^{b}$ Robert S. Eisenberg DD $^{c}$ and \\ David D. L. Minh (D) *a
}

\begin{abstract}
Although ligand-binding sites in many proteins contain a high number density of charged side chains that can polarize small organic molecules and influence binding, the magnitude of this effect has not been studied in many systems. Here, we use a quantum mechanics/molecular mechanics (QM/MM) approach, in which the ligand is the QM region, to compute the ligand polarization energy of 286 protein-ligand complexes from the PDBBind Core Set (release 2016). Calculations were performed both with and without implicit solvent based on the domain decomposition Conductor-like Screening Model. We observe that the ligand polarization energy is linearly correlated with the magnitude of the electric field acting on the ligand, the magnitude of the induced dipole moment, and the classical polarization energy. The influence of protein and cation charges on the ligand polarization diminishes with the distance and is below $2 \mathrm{kcal} \mathrm{mol}^{-1}$ at $9 \AA$ and $1 \mathrm{kcal} \mathrm{mol}^{-1}$ at $12 \AA$. Compared to these embedding field charges, implicit solvent has a relatively minor effect on ligand polarization. Considering both polarization and solvation appears essential to computing negative binding energies in some crystallographic complexes. Solvation, but not polarization, is essential for achieving moderate correlation with experimental binding free energies.
\end{abstract}

\section{Introduction}

Noncovalent binding to proteins is a key mechanism by which small organic molecules (ligands) interact with biological systems. Most drugs are noncovalent inhibitors of particular targets. Signaling molecules generally bind to specific receptors. Molecules with low solubility often bind to serum albumin. Even in enzymes, some noncovalent binding of substrates is a prerequisite to catalysis.

Many proteins generate a strong electrostatic potential that can influence ligand binding. To promote stable folding, globular proteins typically consist of a hydrophobic core and hydrophilic surface. Many amino acids in the latter region are charged. Indeed, in an analysis of 573 enzyme structures, Jimenez-Morales et al. ${ }^{1}$ observed a high number density of oft-charged acidic (aspartic and glutamic acid) and basic (lysine, arginine, and histidine) amino acids in catalytic sites $\left(18.9 \pm 0.58 \mathrm{~mol} \mathrm{~L}^{-1}\right)$ and other surface pockets, including ligand-binding sites $\left(28.2 \pm 0.34 \mathrm{~mol} \mathrm{~L}^{-1}\right)$. For context, the number density of charges is $2.82 \pm 0.03 \mathrm{~mol} \mathrm{~L}^{-1}$ in entire

\footnotetext{
${ }^{a}$ Department of Chemistry, Illinois Institute of Technology, Chicago, Illinois, 60616, USA.E-mail: dminh@iit.edu

${ }^{b}$ Department of Computer Science, Illinois Institute of Technology, Chicago, Illinois, 60616, USA

${ }^{c}$ Department of Applied Mathematics, Illinois Institute of Technology, Chicago, Illinois, 60616, USA

$\dagger$ Electronic supplementary information (ESI) available. See DOI: 10.1039/d0cp00376
}

proteins $^{1}$ and $74.3 \mathrm{~mol} \mathrm{~L}^{-1}$ in a sodium chloride salt crystal. ${ }^{2}$ Charged amino acid side chains generate patterns in the surrounding electrostatic potential that can have functional roles that include mediating associations with other proteins with complementary electrostatics and channeling charged enzyme substrates. ${ }^{3}$ Within a protein, electrostatic forces can alter redox potentials, shift the $\mathrm{p} K_{\mathrm{a}} \mathrm{s}$ of amino acid residues, ${ }^{3}$ accelerate enzyme catalysis, ${ }^{4,5}$ and polarize ligands. ${ }^{6}$

The importance of ligand polarization in protein-ligand binding has been demonstrated by studies that compare results from similar models with and without polarization. Although the vast majority of current studies modeling biological macromolecules are based on fixed-charge molecular mechanics force fields, polarizable models are being actively developed. ${ }^{7,8}$ Jiao et $a l .{ }^{9}$ demonstrated that incorporating polarization into a molecular mechanics force field was essential for accurately computing the binding free energy between trypsin and the charged ligands benzamidine and diazamidine. Quantum mechanics (QM) and mixed quantum mechanics/molecular mechanics (QM/MM) methods have also been increasingly employed in predicting the binding pose - the configuration and orientation of a ligand in a complex - and binding affinity. ${ }^{10,11}$ Semiempirical QM methods have shown particular promise in correctly distinguishing the native (near-crystallographic) binding pose from decoy poses (non-native poses that have low docking scores) in diverse sets of protein-ligand complexes. ${ }^{12-15} \mathrm{QM} / \mathrm{MM}$ methods usually couple the QM and 
MM regions via electrostatic embedding, in which charges from the $\mathrm{MM}$ region alter the Hamiltonian in the QM region. Electrostatic embedding allows the QM region (which in most protein-ligand binding studies includes the ligand and sometimes surrounding residues) to polarize in response to charges in the environment. Cho et al. ${ }^{16}$ demonstrated the importance of embedding by evaluating the ability of multiple docking schemes to recapitulate ligand binding poses in 40 diverse complexes. They found that assigning ligand charges using a QM/MM method with electrostatic embedding was generally more successful than a gas-phase QM method without embedding. Subsequently, Kim and $\mathrm{Cho}^{17}$ performed a more systematic assessment focusing on $40 \mathrm{G}$ protein-coupled receptor crystal structures. The QM/MM method outperformed (1.115 ^ average RMSD and RMSD $<2 \AA$ in 36/40 complexes) a gas-phase QM method without embedding (1.672 $\AA$ average RMSD and RMSD $<2 \AA$ in $31 / 40$ complexes) and a fixed-charge molecular mechanics method (1.735 $\AA$ average RMSD and RMSD $<2 \AA$ in 32/40 complexes). Beyond the context of protein-ligand binding, the inclusion of the polarization energy has been shown to dramatically affect water density ${ }^{18}$ and the structure and dynamics of solvated ions in water clusters. ${ }^{19-22}$

Ligand polarization effects have also been isolated using a decomposition scheme pioneered by Gao and Xia, ${ }^{23}$ which was originally applied to the polarization of solutes by aqueous solvents. In this scheme, the polarization energy of molecule $I$, $\Xi_{I}^{\text {pol }}$ (eqn (6)), is the sum of the energy from distorting the wave function, $\Xi_{I}^{\text {dist }}$ (eqn (8)), and the energy from stabilizing Coulomb interactions relative to the gas phase, $\Xi_{I}^{\text {stab }}$ (eqn (9)). For three high-affinity inhibitors of human immunodeficiency virus type 1 (HIV-1) protease, Hensen et al. ${ }^{6}$ found that the magnitude of the ligand polarization energy can be as large as one-third of the electrostatic interaction energy. Fong et al. ${ }^{24}$ considered 6 ligands of HIV-1 protease in near-native poses and found that depending on the level of theory, the polarization energy is from $16 \%$ to $21 \%$ of the electrostatic interaction energy.

Although comparative studies and energy decomposition schemes have strongly indicated the importance of ligand polarization, the magnitude of this term and the factors contributing to the ligand polarization energy have not, to our knowledge, been investigated for many diverse systems. Moreover, the extent of ligand polarization by the protein environment has not been compared to the extent of ligand polarization by solvent. Here, we address this knowledge gap by calculating the ligand polarization energy, with and without a continuum dielectric implicit solvent model, for 286 proteinligand complexes from the PDBBind Core Set (release 2016). ${ }^{25}$ The PDBBind is a comprehensive database of complexes for which both Protein Data Bank crystal structures and binding affinity data are available. The Core set is a subset of the PDBBind with high-quality and non-redundant structures meant as a benchmark for molecular docking methods. The size and diversity of this dataset allow us to draw more general and statistically meaningful conclusions about ligand polarization than previous efforts. Additionally, calculations with implicit solvent allow us to compare the magnitude of ligand polarization by the protein and the solvent.

\section{Theory and methods}

\subsection{Energies}

We employed a QM/MM scheme in which the ligand is the QM region and other atoms are the $\mathrm{MM}$ region. To enable energy decomposition, the Schrödinger equation for the ligand was solved for several Hamiltonians: in the gas phase, with electrostatic embedding, and with electrostatic embedding and a continuum dielectric implicit solvent model.

In the gas phase, the Hamiltonian operator $\hat{H}_{I}$ of a molecule $I$ is,

$$
\hat{H}_{I}=\sum_{i \in I} \frac{1}{2} \frac{\hat{p}_{i}^{2}}{m_{\mathrm{e}}}+\sum_{i \in I} \sum_{\substack{j>i \\ j \in I}} \frac{1}{r_{i j}}-\sum_{i \in I} \sum_{A \in I} \frac{Z_{A}}{\left|\boldsymbol{r}_{i}-\boldsymbol{R}_{A}\right|}+\sum_{A \in I} \sum_{\substack{B>A \\ B \in I}} \frac{Z_{A} Z_{B}}{R_{A B}},
$$

where $i$ and $j$ are indices over all electrons and $A$ and $B$ are indices over all atoms in molecule $I . \hat{p}_{i}$ is the momentum operator and $m_{\mathrm{e}}$ is the mass of an electron. $\boldsymbol{r}_{i}$ is the position of electron $i, \boldsymbol{R}_{A}$ is the position of atom $A$, and $Z_{A}$ is the atomic number of atom $A$. $r_{i j}$ is the distance between electrons $i$ and $j$, and $R_{A B}$ is the distance between atoms $A$ and $B$. The groundstate energy $E_{I}$ of the molecule $I$ is

$$
E_{I}=\left\langle\Psi_{I}\left|\hat{H}_{I}\right| \Psi_{I}\right\rangle,
$$

where $\Psi_{I}$ is the electronic wave function of the molecule $I$.

When the molecule $I$ is placed in an embedding field $Q_{I}=\left\{q_{F}\right\}$, the Hamiltonian operator of the embedded molecule is given by $\hat{H}_{I: Q_{I}}=\hat{H}_{I}+\hat{H}_{\left[I / Q_{I}\right]}$. We will use $I: Q_{I}$ to denote the embedding of molecule $I$ in the embedding field $Q_{I}$. The Hamiltonian operator $\hat{H}_{\left[I / Q_{I}\right]}$ for Coulomb interactions between the molecule $I(\mathrm{QM})$ and the field $Q_{I}(\mathrm{MM})$ is,

$$
\hat{H}_{\left[I / Q_{I}\right]}=-\sum_{i \in I} \sum_{F \in Q_{I}} \frac{q_{F}}{\left|\boldsymbol{r}_{i}-\boldsymbol{R}_{F}\right|}+\sum_{A \in I} \sum_{F \in Q_{I}} \frac{Z_{A} q_{F}}{\left|\boldsymbol{R}_{A}-\boldsymbol{R}_{F}\right|},
$$

where $F$ is an index over charges in the embedding field. The first summand describes electron-charge interactions and the second proton-charge interactions. The ground-state energy $E_{I: Q_{I}}$ of the embedded molecule $I: Q_{I}$ is obtained by

$$
E_{I: Q_{I}}=\left\langle\Psi_{I: Q_{I}}\left|\hat{H}_{I: Q_{I}}\right| \Psi_{I: Q_{I}}\right\rangle,
$$

where $\Psi_{I: Q_{I}}$ is the ground-state electronic wave function of the embedded molecule $I: Q_{I}$. The embedding field should affect the ground-state wave function of the molecule such that $\left|\Psi_{I: Q_{I}}\right|^{2} \neq$ $\left|\Psi_{I}\right|^{2}$.

We will use the symbol $\Xi$ to denote a difference between two expectation values. The electronic interaction energy describes the change in electronic energy of a molecule upon interaction with the embedding field,

$$
\Xi_{I}^{\text {elec }}=\left\langle\Psi_{I: Q_{I}}\left|\hat{H}_{I: Q_{I}}\right| \Psi_{I: Q_{I}}\right\rangle-\left\langle\Psi_{I}\left|\hat{H}_{I}\right| \Psi_{I}\right\rangle
$$


Hensen $e t a l .{ }^{6}$ decomposed $\Xi_{I}^{\text {elec }}$ into the polarization energy of a molecule,

$$
\Xi_{I}^{\mathrm{pol}}=\left\langle\Psi_{I: Q_{I}}\left|\hat{H}_{I: Q_{I}}\right| \Psi_{I: Q_{I}}\right\rangle-\left\langle\Psi_{I}\left|\hat{H}_{I: Q_{I}}\right| \Psi_{I}\right\rangle,
$$

the difference in the expectation of $\hat{H}_{I: Q_{I}}$ between the gas phase and in the embedding field, and the Coulomb interaction energy between a molecule and the embedding field,

$$
E_{I: Q_{I}}^{\mathrm{Coul}}=\left\langle\Psi_{I}\left|\hat{H}_{\left[I / Q_{I}\right]}\right| \Psi_{I}\right\rangle .
$$

such that $\Xi_{I}^{\text {elec }}=\Xi_{I}^{\mathrm{pol}}+E_{I: Q_{I}}^{\text {Coul }}$. Hensen et al. ${ }^{6}$ further decomposed the polarization energy $\Xi_{I}^{\text {pol }}$ into an energy of distorting the gas-phase wave function,

$$
\Xi_{I}^{\text {dist }}=\left\langle\Psi_{I: Q_{I}}\left|\hat{H}_{I}\right| \Psi_{I: Q_{I}}\right\rangle-\left\langle\Psi_{I}\left|\hat{H}_{I}\right| \Psi_{I}\right\rangle,
$$

and the energy of stabilizing interactions with the embedding field $Q_{I}=\left\{q_{F}\right\}$,

$$
\Xi_{I}^{\mathrm{stab}}=\left\langle\Psi_{I: Q_{I}}\left|\hat{H}_{\left[I / Q_{I}\right]}\right| \Psi_{I: Q_{I}}\right\rangle-\left\langle\Psi_{I}\left|\hat{H}_{\left[I / Q_{I}\right]}\right| \Psi_{I}\right\rangle .
$$

In a system of $N$ molecules, the total electronic interaction energy and its decomposition into polarization and permanent Coulomb energies are,

$$
\begin{gathered}
\Xi^{\text {elec }}=\Xi^{\mathrm{pol}}+E^{\mathrm{Coul}} \\
\Xi^{\mathrm{pol}}=\frac{1}{2} \sum_{I} \Xi_{I}^{\mathrm{pol}} \\
E^{\mathrm{Coul}}=\frac{1}{2} \sum_{I} E_{I: Q_{I}}^{\text {Coul }} .
\end{gathered}
$$

In both polarization and Coulomb energies, $\Xi^{\mathrm{pol}}$ and $E^{\mathrm{Coul}}$, the factor of $1 / 2$ is introduced to compensate for doubly counting the interaction energy. Like $\Xi^{\mathrm{pol}}, \Xi^{\mathrm{dist}}$, and $\Xi^{\mathrm{stab}}$ are similarly defined.

In our present scheme, only one molecule, the ligand, is treated quantum mechanically. Thus $\Xi^{\text {elec }}=\Xi_{I}^{\text {elec }}, \Xi^{\mathrm{pol}}=\Xi_{I}^{\mathrm{pol}}$, $\Xi^{\text {dist }}=\Xi_{I}^{\text {dist }}, \Xi^{\text {stab }}=\Xi_{I}^{\text {stab }}$, and $E^{\text {Coul }}=E_{I: Q_{I}}^{\text {Coul }}$, where $I$ is the ligand molecule.

Both $\Psi_{I}$ and $\Psi_{I: Q_{I}}$ were calculated using the restricted Hartree-Fock method ${ }^{26}$ in conjunction with the $6-311 \mathrm{G}^{* *}$ basis set. $^{27}$ The atomic charge of atoms $A$ with and without the embedding field $Q_{I}=\left\{q_{F}\right\}, q_{A}^{\mathrm{QM}: Q_{I}}$ and $q_{A}^{\mathrm{QM}}$, respectively, were obtained by fitting to the quantum mechanical electrostatic potential (ESP) using the restrained electrostatic potential (RESP) method. ${ }^{28}$ Fitted point charges were used to evaluate the stabilization energy,

$$
\Xi^{\mathrm{stab}}=\sum_{A \in I} \sum_{F \in Q_{I}}\left(q_{A}^{\mathrm{QM}: Q_{I}}-q_{A}^{\mathrm{QM}}\right) \frac{q_{F}}{R_{A F}} .
$$

For most reported calculations, the embedding field $Q_{I}=\left\{q_{F}\right\}$ consisted of all of the non-ligand atoms in the system. In order to evaluate the distance at which embedding field atoms affect the polarization energy, we also performed calculations in which the embedding field consists of all atoms within a cutoff parameter $R_{\text {cut }}$ of any ligand atom. The cutoff parameter was varied from $R_{\text {cut }} \in\{4,5, \ldots, 10,12, \ldots, 20\}$. Even when different
$R_{\text {cut }}$ were used for determining $\Psi_{I: Q_{I}}$ and $q_{A}^{\mathrm{QM}: Q_{I}}$, energies were evaluated using an embedding field based on all atoms in the model.

In addition to the electrostatic interaction energy, coupling between the QM and MM region also includes a van der Waals interaction energy modeled by the Lennard-Jones potential,

$$
E^{\mathrm{vdW}}=\sum_{A \in I} \sum_{F \in Q_{I}} 4 \varepsilon_{A F}\left[\left(\frac{\sigma_{A F}}{R_{A F}}\right)^{12}-\left(\frac{\sigma_{A F}}{R_{A F}}\right)^{6}\right],
$$

where $\sigma_{\mathrm{AF}}$ and $\varepsilon_{\mathrm{AF}}$ are the Lennard-Jones parameters. Combined with $E^{\mathrm{Coul}}, E^{\mathrm{vdw}}$ makes up the intermolecular pairwise interaction energy,

$$
E^{\mathrm{pair}}=E^{\mathrm{Coul}}+E^{\mathrm{vdW}} .
$$

Because we are interested in molecules in solution, opposed to the gas phase, we also consider the solvation free energy. We will use $W(X)$ to denote a solvation free energy, where $X \in\{\mathrm{PL}, \mathrm{P}, \mathrm{L}\}$ represent the complex, protein, and ligand, respectively. The solvation free energy is an integral over all the solvent degrees of freedom. There are many possible ways to compute this quantity. In this paper, we used two continuum dielectric implicit solvent models for the electrostatic component of the solvation free energy: the Onufriev Bashford Case 2 $(\mathrm{OBC} 2)^{29}$ generalized Born and domain decomposition COnductorlike Screening MOdel (ddCOSMO). ${ }^{30,31}$ We will elaborate on how we applied these models in subsequent paragraphs, but here we would like to point out a key distinction between the models: ddCOSMO accounts for polarization of the solute by solvent. On the other hand, the OBC2 model, which was developed for fixed-charge molecular mechanics force fields, does not. The nonpolar cavity formation term in the solvation free energy was calculated as the product of the surface tension $\gamma$ and surface area $A(X)$.

Given a particular solvation free energy model, the total binding energy is given by (Fig. 1),

$$
\begin{gathered}
\Psi^{\text {bind }}=E^{\text {pair }}+\Xi^{\text {pol }}+W^{\text {bind }}, \\
W^{\text {bind }}=W(\mathrm{PL})-W(\mathrm{P})-W(\mathrm{~L}) .
\end{gathered}
$$

As these energy terms - $\Psi^{\text {bind }}, W^{\text {bind }}$, and $W$-are dependent on the solvation energy model, we will use a subscript to describe the model, e.g. $\Psi_{\mathrm{OBC} 2}^{\mathrm{bind}}$.

In applying the OBC2 model, which does not explicitly account for polarization by solvent, we used different ligand partial charge schemes. In the $W_{\mathrm{OBC} 2}(\mathrm{PL})$ calculation, $q_{A}^{\mathrm{QM}: Q_{I}}$ are used for ligand partial charges. On the other hand, the $W_{\mathrm{OBC} 2}(\mathrm{~L})$ calculation uses $q_{A}^{\mathrm{QM}}$ for ligand partial charges. To isolate the effects of polarization by the embedding field, we also define a total binding energy that does not consider ligand polarization,

$$
\begin{gathered}
\Psi_{\mathrm{OBC} 2}^{\text {bind,np }}=E^{\text {pair }}+W^{\text {bind,np }}, \\
W_{\mathrm{OBC} 2}^{\text {bind,np }}=W(\mathrm{PL}, n p)-W(\mathrm{P})-W(\mathrm{~L}) .
\end{gathered}
$$




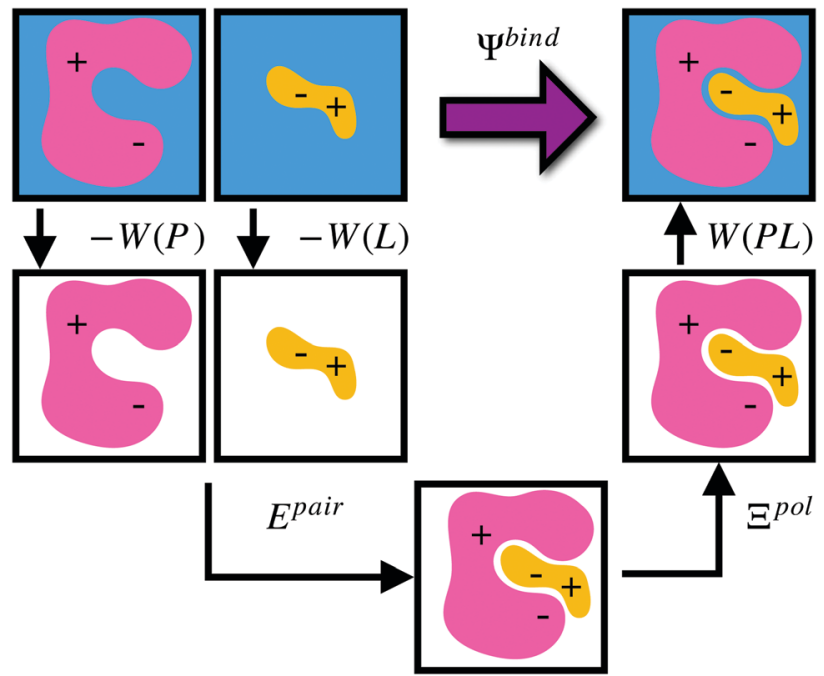

Fig. 1 Schematic illustrating the decomposition of binding energy, $\Psi^{\text {bind }}$ into desolvation free energy of the protein, $-W(\mathrm{P})$, the desolvation free energy of the ligand, $-W(L)$, the intermolecular pairwise interaction energy, $E^{\text {pair }}$, the ligand polarization energy, $\Xi^{\text {pol }}$, and the solvation free energy of the complex, $W(\mathrm{PL})$.

$W_{\mathrm{OBC} 2}^{\mathrm{bind}, \mathrm{np}}$ differs from $W_{\mathrm{OBC} 2}^{\mathrm{bind}}$ because $q_{A}^{\mathrm{QM}}$ are used for ligand partial charges in the $W(\mathrm{PL})$ calculation. $\Psi_{\mathrm{OBC} 2}^{\mathrm{bind}, \mathrm{np}}$ is the binding energy for a purely MM model.

In the COnductor-like Screening MOdel (COSMO), the solvent is treated as a set of apparent charges on the surface of the solute cavity. These charges interact with the system according to Coulomb's law, such that,

$$
\hat{H}_{[I / \mathrm{sol}]}=\frac{f(\varepsilon)}{2}\left[-\sum_{i \in I} \sum_{s \in \Gamma} \frac{q_{s}}{\left|\boldsymbol{r}_{i}-\boldsymbol{R}_{s}\right|}+\sum_{A \in I} \sum_{s \in \Gamma} \frac{Z_{A} q_{s}}{\left|\boldsymbol{R}_{A}-\boldsymbol{R}_{s}\right|}\right],
$$

where $q_{s}$ is an apparent surface charge (ASC) on the surface $\Gamma$ of the cavity and $f(\varepsilon)=(\varepsilon-1) / \varepsilon$ is an empirical scaling depending on the solvent dielectric constant $\varepsilon$ used to take into account the nonconductor nature of the solvent. The dielectric constant of water is $\varepsilon=78.3553$. The ground state energy $E_{I, \text { sol }}$ and electronic wave function $\Psi_{I \text {,sol }}$ of the molecule is obtained as the solution to the Schrödinger equation with the Hamiltonian operator $\hat{H}_{I \text {,sol }}=\hat{H}_{I}+\hat{H}_{[I / \mathrm{sol}]}$ that is also consistent with the ASC. The Hamiltonian for the interaction between the molecule in an embedding field with the apparent surface charges, $\hat{H}_{\left[I: Q_{I} / \text { sol }\right]}$, can be defined analogously. The ground state energy $E_{I: Q_{I}}$,sol and electronic wave function $\Psi_{I: Q_{I} \text {,sol }}$ of the embedded molecule is also obtained as the solution to the Schrödinger equation with the Hamiltonian operator $\hat{H}_{I: Q_{I}, \text { sol }}=\hat{H}_{I: Q_{I}}+\hat{H}_{\left[I: Q_{I} / \text { sol }\right]}$. In the case of the molecule in the embedding field, the region enclosed by the surface not only includes the atoms in the molecule but also embedding field atoms. $\hat{H}_{\left[I: Q_{I} / \mathrm{sol}\right]}$ also includes interactions between embedding field charges and the apparent surface charge. In our calculations, the surface of the cavity was defined as the solvent-accessible surface $e^{32,33}$ based on the van der Waals radii ${ }^{34}$ scaled by a factor $1.2 .^{35}$ The ASCs $\left(\left\{q_{s}\right\}\right)$ on the surface $\Gamma$ were estimated using the domain decomposition COnductor-like Screening MOdel
(ddCOSMO) scheme. $^{30,31}$ Unlike COSMO, ddCOSMO scales linearly with the number of ACSs, a particularly beneficial feature in systems with large surfaces, such as proteins.

For a species $\mathrm{X}$ in ddCOSMO solvent, the solvation free energy is given by

$$
W_{\mathrm{ddCOSMO}}(X)=\Xi_{X, \mathrm{sol}}^{\mathrm{elec}}+\Delta G_{\mathrm{surf}},
$$

where $X \in\{\mathrm{PL}, \mathrm{P}, \mathrm{L}\}$ represent the complex, protein, and ligand. $\Delta G_{\text {surf }}$ is the nonpolar component of the solvation free energy and was estimated based on the ACE surface area approximation. ${ }^{36}$

The electrostatic components of these energies are given by,

$$
\Xi_{X, \text { sol }}^{\text {elec }}=\left\langle\Psi_{X, \text { sol }}\left|\hat{H}_{X, \text { sol }}\right| \Psi_{X, \text { sol }}\right\rangle-\left\langle\Psi_{X}\left|\hat{H}_{X}\right| \Psi_{X}\right\rangle .
$$

As with the effect of the embedding field, these electronic interaction energies may be further decomposed into a polarization energy and Coulomb interaction energy,

$$
\begin{gathered}
\Xi_{X, \mathrm{sol}}^{\mathrm{pol}}=\left\langle\Psi_{X, \mathrm{sol}}\left|\hat{H}_{Y, \mathrm{sol}}\right| \Psi_{X, \mathrm{sol}}\right\rangle-\left\langle\Psi_{X}\left|\hat{H}_{Y, \mathrm{sol}}\right| \Psi_{X}\right\rangle, \\
E_{X, \mathrm{sol}}^{\mathrm{Coul}}=\left\langle\Psi_{X}\left|\hat{H}_{[Y / \mathrm{sol}]}\right| \Psi_{X}\right\rangle,
\end{gathered}
$$

where $Y$ is dependent on $X$. With the ligand, $X=\mathrm{L}$ and likewise $Y=\mathrm{L}$ is the molecule itself. With the protein, $\Xi_{\mathrm{P}, \mathrm{sol}}^{\mathrm{pol}}=0$ because the protein atoms are not polarizable in our scheme. With the complex, $X=\mathrm{PL}$ and $Y=\mathrm{L}: \mathrm{P}$, the ligand in the embedding field of protein charges.

A helpful way to summarize the effect of polarization by the solvent (in addition to polarization by the embedding field) is

$$
\Xi_{\mathrm{sol}}^{\mathrm{pol}}=\Xi_{\mathrm{L}, \mathrm{sol}}^{\mathrm{pol}}-\Xi_{\mathrm{L}, \mathrm{sol}}^{\mathrm{pol}}
$$

\subsection{Other properties}

We computed a number of other properties to assess whether they have a clear relationship with the polarization energy.

Motivated by the observation of a high density of acid and base side chains in enzymes, ${ }^{1}$ we computed two quantities: the percentage of atoms in a protein that are highly charged; and the number density of highly charged atoms within $6 \AA$ of any ligand atom. The percentage of atoms in the protein that are highly charged is defined as,

$$
\frac{1}{N} \sum_{i}^{N} H\left(\left|q_{i}\right|-0.6\right) \times 100,
$$

where $i$ is an index over atoms in the protein and $N$ is the total number of atoms in the protein. This expression uses the Heaviside step function,

$$
H(x)= \begin{cases}0, & x<0 \\ 1, & x \geq 0\end{cases}
$$

where $x$ is a real number. The volume of the binding site was determined by Monte Carlo integration. To perform this integration, a box was defined that includes $6 \AA$ around the range of the ligand atoms in each dimension. Points within the box were randomly sampled from a uniform distribution and assessed for the distance to the nearest ligand atom. The site volume was 
estimated by the product of the box volume and the fraction of points in the box within $6 \AA$ of a ligand atom.

We also computed a number of properties inspired by classical electrostatics. In classical electrostatics, the internal energy of a dipole moment in an electric field is the dot product of the dipole with the field. We considered two classical models: one in which the entire ligand is treated as a dipole and a second in which each atom is treated as a dipole.

If the ligand is considered as a dipole, the change in internal energy due to an induced dipole is,

$$
\Xi^{\mathrm{pol}, \mathrm{cL}}=-\mu_{\mathrm{L}}^{\mathrm{ind}} \cdot \mathbf{E}_{\mathrm{L}}^{0},
$$

where $\mu_{\mathrm{L}}^{\text {ind }}$ is the induced dipole moment of the ligand $\mathrm{L}$ and $\mathbf{E}_{\mathrm{L}}^{0}$ is the electric field acting on the ligand $\mathrm{L}$ due to the embedding field $Q_{\mathrm{L}}=\left\{q_{F}\right\}$ consisting of atomic charges of the surrounding atoms. The electric field acting on the center of mass (or protons) $\boldsymbol{R}_{C}$ of the ligand is,

$$
\mathbf{E}_{\mathrm{L}}^{0}=\sum_{F \in Q_{\mathrm{L}}} \frac{q_{F}}{R_{C F}{ }^{3}} \boldsymbol{R}_{C F},
$$

where $F$ runs over the atomic sites in the embedding field. $\boldsymbol{R}_{C F}=\boldsymbol{R}_{C}-\boldsymbol{R}_{F}$ and $R_{C F}=\left|\boldsymbol{R}_{C F}\right|$.

The induced dipole moment of the ligand $\mu_{\mathrm{L}}^{\text {ind }}$ was calculated in two ways. The first was from the expectation value of the dipole moments,

$$
\begin{aligned}
\mu_{\mathrm{L}}^{\text {ind,QM }} & =\left\langle\Psi_{\mathrm{L}: Q_{\mathrm{L}}}|\hat{\mu}| \Psi_{\mathrm{L}: Q_{\mathrm{L}}}\right\rangle-\left\langle\Psi_{\mathrm{L}}|\hat{\mu}| \Psi_{\mathrm{L}}\right\rangle \\
& =\boldsymbol{\mu}_{\mathrm{L}}^{\mathrm{QM}: Q_{L}}-\boldsymbol{\mu}_{\mathrm{L}}^{\mathrm{QM}},
\end{aligned}
$$

where $\hat{\mu}$ is the dipole moment operator. The second was based on the molecular polarizability tensor, $\boldsymbol{\alpha}_{\mathrm{L}}$, and the electric field on the center of mass of the ligand,

$$
\mu_{\mathrm{L}}^{\text {ind, } \alpha_{\mathrm{L}}}=\alpha_{\mathrm{L}} \mathbf{E}_{\mathrm{L}}^{0} .
$$

Elements of the molecular polarizability tensor $\left(\boldsymbol{\alpha}_{\mathrm{L}}\right)_{x y}$ describe the susceptibility of a molecule to polarization along the $x$ axis due to an electric field along the $y$ axis. As in Willow et al., ${ }^{37}$ these tensor elements were calculated based on placing a pair of point charges of $\mp 1$ a.u. at $\boldsymbol{R}_{\mathrm{cm}} \pm 100$ Bohr along a Cartesian axis, where $\boldsymbol{R}_{\mathrm{cm}}$ represents the center of mass of the ligand, to create an electric field. Then $\left(\boldsymbol{\alpha}_{\mathrm{L}}\right)_{x y}$ were evaluated as the ratio of the induced dipole moment due the point charges, $\mu_{\mathrm{L}}^{\text {ind,pc }}$, and the electric field applied by the point charge onto the ligand, $\mathbf{E}_{\mathrm{L}}^{0, \mathrm{pc}}$,

$$
\left(\boldsymbol{\alpha}_{\mathrm{L}}\right)_{x y}=\frac{\left(\boldsymbol{\mu}_{\mathrm{L}}^{\text {ind,pc }}\right)_{x}}{\left(\mathbf{E}_{\mathrm{L}}^{0, \mathrm{pc}}\right)_{y}} .
$$

The dipole moment from the electron density is more accurate and valuable for assessing the correspondence between $\Xi^{\mathrm{pol}}$ and $\Xi^{\text {pol,c }}$. However, it is not a practical shortcut to the polarization energy because it requires the same quantum chemistry calculations used to compute $\Xi^{\mathrm{pol}}$. On the other hand, although the molecular polarizability tensor, $\boldsymbol{\alpha}_{\mathrm{L}}$, requires three quantum chemistry calculations, it can be reused (as an approximation) for multiple ligand configurations. Hence, the dipole moment from the molecular polarizability tensor, $\mu_{\mathrm{L}}^{\text {ind }, \alpha_{\mathrm{L}}}$, could potentially reduce the computational costs of $\Xi^{\text {pol }}$ prediction. To facilitate comparison with the polarization energy, we also computed the molecular polarizability scalar of the ligand, $\alpha_{\mathrm{L}}$, defined as,

$$
\alpha_{\mathrm{L}}=\frac{1}{3} \operatorname{Tr}\left[\boldsymbol{\alpha}_{\mathrm{L}}\right],
$$

where $\operatorname{Tr}$ is the trace of a square matrix.

If each atom on the ligand is considered as a dipole, then the change in internal energy due to an induced dipole is,

$$
\Xi^{\mathrm{pol}, \mathrm{cA}}=-\sum_{A \in L} \boldsymbol{\mu}_{A}^{\mathrm{ind}} \cdot \mathbf{E}_{A}^{0},
$$

The electric field acting on an atom is,

$$
\mathbf{E}_{A}^{0}=\sum_{F \in Q_{\mathrm{L}}} \frac{q_{F}}{R_{A F}{ }^{3}} \boldsymbol{R}_{A F},
$$

where $A$ runs over all atomic sites in the ligand. The induced dipole on each atom was computed based on RESP charges as,

$$
\boldsymbol{\mu}_{A}^{\mathrm{ind}}=\left(q_{A}^{\mathrm{QM}: Q_{\mathrm{L}}}-q_{A}^{\mathrm{QM}}\right) \boldsymbol{R}_{A} .
$$

In all, we considered the relationship between $\Xi^{\mathrm{pol}}$ and a number of other properties: the

(1) percentage of highly charged atoms in a protein (eqn (26));

(2) molecular polarizability scalar, $\alpha_{\mathrm{L}}$ (eqn (33));

(3) Coulomb interaction energy, $E^{\text {Coul }}$ (eqn (7));

(4) magnitude of the electric field on the ligand center of mass, $\left|\mathbf{E}_{\mathrm{L}}^{0}\right|$, where $\mathbf{E}_{\mathrm{L}}^{0}$ is from eqn (29);

(5) magnitude of total electric field on the ligand atom sites, $\left|\sum_{A \in L} \mathbf{E}_{A}^{0}\right|$, where $\mathbf{E}_{A}^{0}$ is from eqn (35);

(6) magnitude of the induced dipole moment based on wave functions, $\left|\mu_{\mathrm{L}}^{\mathrm{ind}, \mathrm{QM}}\right|$, where $\boldsymbol{\mu}_{\mathrm{L}}^{\mathrm{ind}, \mathrm{QM}}$ is from eqn (30);

(7) magnitude of the induced dipole moment based on the molecular polarizability tensor, $\left|\boldsymbol{\mu}_{\mathrm{L}}^{\text {ind, }, \alpha_{\mathrm{L}}}\right|$, where $\boldsymbol{\mu}_{\mathrm{L}}^{\text {ind, }, \alpha_{\mathrm{L}}}$ is from eqn (31);

(8) classical polarization energy of a ligand dipole, $\Xi^{\mathrm{pol,cL}}$ (eqn (28)), using eqn (30) for the induced dipole moment;

(9) classical polarization energy of a ligand dipole, $\Xi^{\mathrm{pol}, \mathrm{cL}, \alpha_{\mathrm{L}}}$ (eqn (28)), using eqn (31) for the induced dipole moment;

(10) classical polarization energy of atomic dipoles, $\Xi^{\mathrm{pol}, \mathrm{cA}}$ (eqn (34));

(11) and ligand polarization energy including solvent effects, $\Xi_{\text {sol }}^{\text {pol }}($ eqn (25)).

\subsection{Computational methods}

Structures from the PDBBind Core Set (release 2016) were processed through an automated workflow based on AmberTools $17^{38}$ and customized QM/MM codes. Protein protonation states were assigned using PDB2PQR 1.9.0 at a pH of 7.0 and ligand protonation states using pkatyper in the QUACPAC 1.7.0.2 toolkit (OpenEye). AMBER topology files based on protein and cation parameters $\left(\mathrm{Na}^{+}, \mathrm{Mg}^{2+}, \mathrm{Ca}^{2+}\right.$, and $\left.\mathrm{Zn}^{2+}\right)$ from 
the AMBER ff14SB force field ${ }^{39}$ and ligand parameters from the Generalized AMBER Force Field $2^{40}$ were built using AmberTools $17 . .^{38}$

Using OpenMM 7.3.1, ${ }^{41}$ complexes in $\mathrm{OBC}^{29}$ generalized Born/surface area implicit solvent were minimized with heavy atom restraints of $2 \mathrm{kcal} \mathrm{mol}^{-1} \AA^{-2}$ towards crystallographic positions until energies converged within $0.24 \mathrm{kcal} \mathrm{mol}^{-1}$.

In our modified QM/MM codes, the evaluation of molecular integrals of many-body operators over Gaussian functions were obtained using libint 2.5.0 ${ }^{42}$ and the linear algebra and eigenvalue decomposition of a symmetric matrix were done with the Armadillo 8.500.1. 43,44 $^{4}$

OpenMM 7.3.1 ${ }^{41}$ was also used to evaluate van der Waals and solvation energies, the latter with the $\mathrm{OBC} 2{ }^{29}$ generalized Born/surface area implicit solvent model. It was also used for the $\Delta G_{\text {surf }}$ term in eqn (21). The PySCF 1.7.0 python package ${ }^{45}$ was used to perform QM/MM calculations in the ddCOSMO scheme. $^{30,31}$

\section{Results and discussion}

\subsection{The distribution of polarization energy is broad and skewed}

Signs of the calculated polarization energy $\Xi^{\text {pol }}$, the distortion energy $\Xi^{\text {dist }}$, and the stabilization energy $\Xi^{\text {stab }}$ are mostly as expected (Fig. 2). In nearly all of the calculations, $\Xi^{\text {pol }}<0$, $\Xi^{\text {dist }}>0$, and $\Xi^{\text {stab }}<0$. The embedding field reshapes the wave function to have stronger Coulomb interactions between the electronic probability density and point charges, such that $\Xi^{\text {stab }}<0$. Because the gas-phase wave function of the ligand has the optimal intramolecular potential, perturbing the wave function leads to a higher intramolecular potential energy such that $\Xi^{\text {dist }}>0$. In the vast majority of systems, the calculated distortion is more than compensated for by the calculated stabilization such that the calculated net effect on the interaction energy due to polarization, $\Xi^{\mathrm{pol}}$, is negative.

Exceptions to the trend of negative calculated ligand polarization energies are due to structural modeling issues that lead to short intermolecular distances. Positive $\Xi^{\text {pol }}$ values were calculated in three complexes. In our models of these structures, there are very short distances between a hydrogen atom in the ligand and in the protein: $0.73 \AA$ in $2 \mathrm{fxs}, 1.06 \AA$ in $3 \mathrm{u} 5 \mathrm{j}$, and $0.87 \AA$ in $4 \mathrm{f} 2 \mathrm{w}$. The close proximity of atoms leads to a severe distortion in the wave function that is not overcome by more favorable Coulomb interactions. These steric clashes could be resolved by changing the models in minor ways that are equally compatible with crystallographic evidence and $\mathrm{p} K_{\mathrm{a}}$ predictions. In the $2 \mathrm{fxs}$ and $4 \mathrm{f} 2 \mathrm{w}$ models, the proton on a carboxylic acid was arbitrarily placed near a ligand hydrogen instead of on the other carbonyl oxygen. In the 3u5j model, the clash could be resolved by switching the position of the terminal oxygen and amine groups, which have nearly identical electron density, on asparagine 140.

The distribution of $\Xi^{\text {pol }}, \Xi^{\text {dist }}$, and $\Xi^{\text {stab }}$ is broad and skewed. There is a peak in the distribution of $\Xi^{\text {pol }}$ around
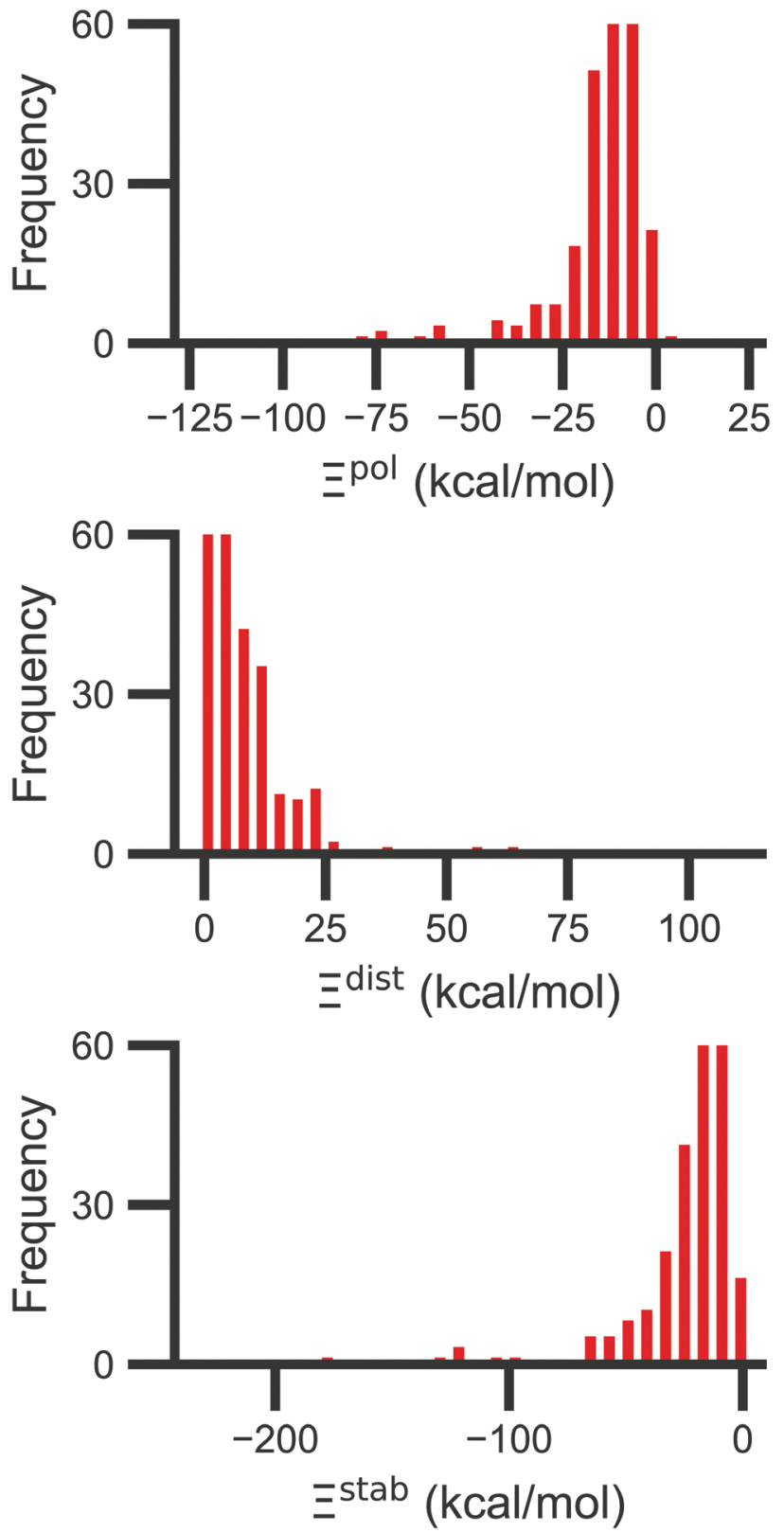

Fig. 2 Histograms of the ligand polarization (top, $\Xi^{\text {pol}}$ ), distortion (middle, $\Xi^{\text {dist }}$ ), and stabilization (bottom, $\Xi^{\text {stab }}$ ) energies in the PDBBind Core Set. The three quantities are related by $\Xi^{\text {pol }}=\Xi^{\text {dist }}+\Xi^{\text {stab }}$.

$-10 \mathrm{kcal} \mathrm{mol}{ }^{-1}$. However, for a small number of complexes, $\Xi^{\mathrm{pol}}$ is much lower, with a minimum value of $-128 \mathrm{kcal} \mathrm{mol}^{-1}$.

\subsection{Systems with the lowest $\Xi^{\text {pol }}$ have close cations}

We hypothesized that the lowest $\Xi^{\text {pol }}$ could be due to crystallographic cations. To test this hypothesis, we subdivided the PDBBind Core Set into two subsets: 90 complexes with cations $\left(\mathrm{Na}^{+}, \mathrm{Mg}^{2+}, \mathrm{Ca}^{2+}\right.$, and $\left.\mathrm{Zn}^{2+}\right)$ and 196 complexes without cations in the crystal structure.

Histograms of $\Xi^{\text {pol }}$ for the two subsets are consistent with our hypothesis (Fig. S1 in the ESI $\dagger$ ). All systems in which $\Xi^{\text {pol }}<$ $-50 \mathrm{kcal} \mathrm{mol}^{-1}$ are in the subset with cations. In contrast, the 
minimum $\Xi^{\text {pol }}$ in the subset without cations is around $-40 \mathrm{kcal} \mathrm{mol}^{-1}$. The range of $\Xi^{\text {dist }}$ and $\Xi^{\text {stab }}$ is also much smaller in the subset without cations.

Crystallographic cations may have an outsize role in ligand polarization because the magnitude of their charge is larger than the charge of most protein atoms. In the AMBER ff14SB force field, ${ }^{39}$ protein partial charges were determined by applying $\mathrm{RESP}^{28}$ to electrostatic potentials from QM calculations. Most protein atoms have near-zero charge. The magnitude of the charge is greater than $0.6 e$, where $e$ represents the elementary charge, in only a few atoms. It is less than $1 e$ in all atoms. These conclusions are also true for protein atoms in our data set (Fig. S2 in the ESI $\dagger$ ). The low magnitude of charge results from delocalization of net charges across several atoms. In contrast, the cations have a charge of $+1 e$ or $+2 e$ that is localized onto a single atom and have a more focused effect on the electrostatic potential.

Beyond the presence of cations, the distance between ligand and cation atoms also plays an important role in ligand polarization (Fig. 3). Even if cations are present in a crystal structure, they are not necessarily close enough to the ligand to significantly polarize its wave function. In many systems,
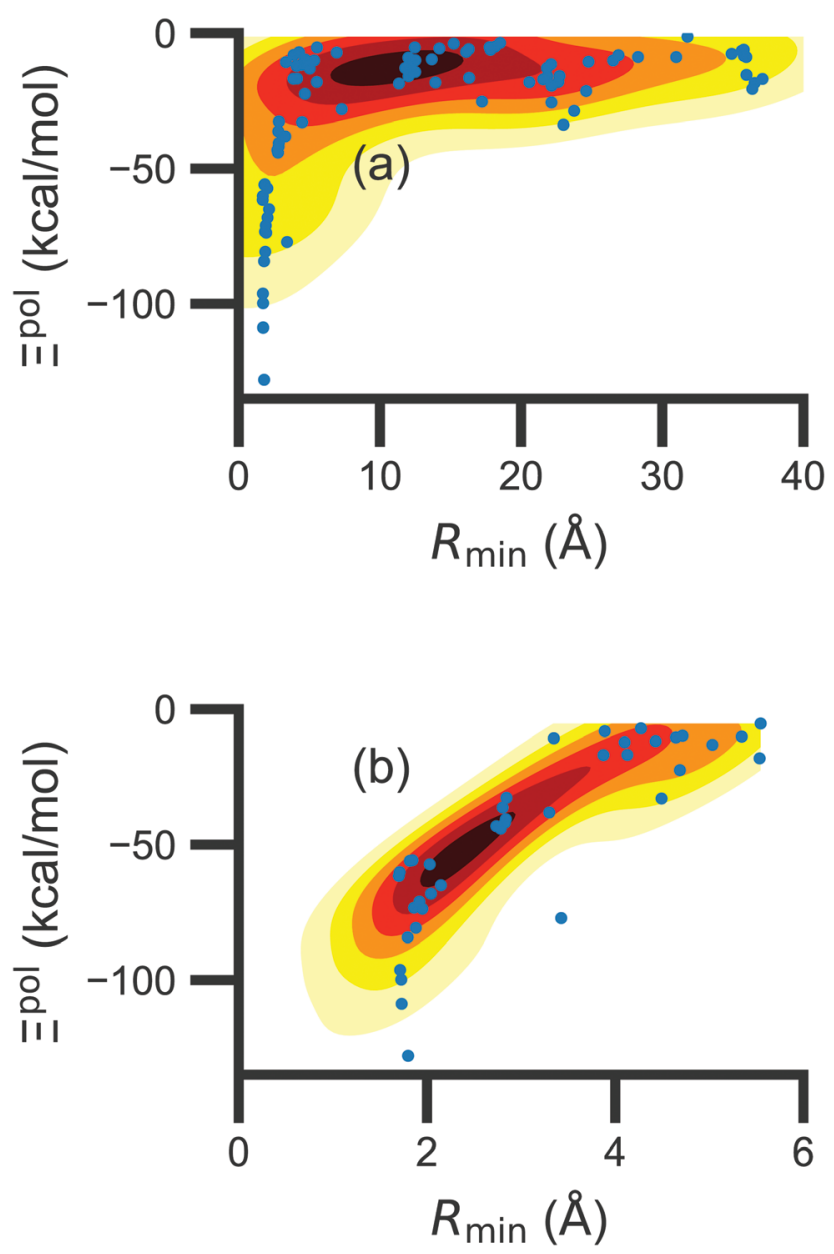

Fig. 3 Scatter plot of the ligand polarization energy $\Xi^{\text {pol }}$ as a function of the minimum distance between a ligand and cation atom, $R_{\min }$, for (a) the entire range of $R_{\min }$ and (b) $R_{\min }<6 \AA$. cations are over $10 \AA$ from any ligand atom. In all of the complexes in which $\Xi^{\text {pol }}<-50 \mathrm{kcal} \mathrm{mol}^{-1}$, a cation is within $4 \AA$ of a ligand atom.

Unfortunately, the extent of ligand polarization when ligands are close to cations is likely overestimated by our $\mathrm{QM} / \mathrm{MM}$ scheme. Because only the ligand is included in the QM region, cations are simply represented as positive point charges. While actual cations have inner-shell electrons that repel further electron density, the point charges are purely attractive. The purely attractive forces draw an unrealistic amount of electron density between the ligand and cation, leading to a very negative polarization energy. For an estimate of the extent of overpolarization in several systems, see Table S1 in the ESI. $\dagger$ As an illustrative example, there is a significant gain in the electron density between the ligand and cation in the complex $3 \mathrm{dx} 1$ (Fig. 4). Hence, we will proceed with extra caution in interpreting points where $\Xi^{\text {pol }}<-50 \mathrm{kcal} \mathrm{mol}^{-1}$.

\subsection{The importance of the embedding field size diminishes with distance}

The size of the embedding field strongly affects estimates of the polarization energy (Fig. 5). Changes in the cutoff distance $R_{\text {cut }}$ alter the partial charges included in the embedding field, the wave function $\Psi_{I: Q_{I}}$, and then the RESP charges. Regardless of $R_{\text {cut }}$, nearly every estimate of $\Delta \Xi^{\mathrm{pol}}\left(R_{\text {cut }}\right)=\Xi^{\mathrm{pol}}\left(R_{\text {cut }}\right)-$ $\Xi^{\mathrm{pol}}\left(R_{\text {cut }}=\infty\right)$ is positive, indicating that the ligand wave function accommodates even distant charges in the embedding field. However, the influence of protein and cation charges diminishes with distance. Correspondingly, as $\Delta \Xi^{\mathrm{pol}}$ diminishes, so does its variance. For larger values of $R_{\text {cut }}=8,9,10$, and $12 \AA$, the mean (and standard deviation) of $\Delta \Xi^{\mathrm{pol}}$ is 1.81 (1.77), 1.49 (1.80), 1.10 (1.23), and 0.92 (1.14) kcal mol ${ }^{-1}$, respectively.

The decomposition of the polarization energy into $E^{\text {Coul }}$ and $E^{\text {dist }}$ is more sensitive to $R_{\text {cut }}$ than the polarization energy itself; distributions of the values (relative to values with no cutoff) and numerical derivatives are broader. Even at $R_{\text {cut }}=8,9,10$, and $12 \AA$, the mean (and standard deviations) of $\Delta E^{\mathrm{Coul}}$ are -2.28 (4.94), -1.46 (4.71), -1.18 (4.06), and -0.99 (3.38) kcal mol $\mathrm{k}^{-1}$.

On average, the decay of $\Delta \Xi^{\text {pol }}$ is well-described by an inverse square law. A nonlinear least-squares regression using

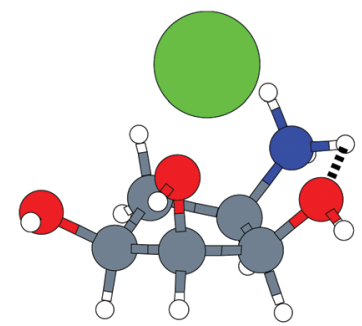

(a)

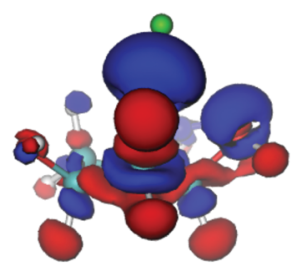

(b)
Fig. 4 (a) The molecular structure of the ligand with one zinc cation $\mathrm{Zn}^{2+}$ in the complex $3 d x 1$. Hydrogen, carbon, nitrogen, oxygen, and zinc atoms are colored with white, gray, blue, red, and green, respectively. (b) The difference in the electronic probability density is plotted. Blue and red contours illustrate the gain and loss of the electronic probability density due to the embedding field. 

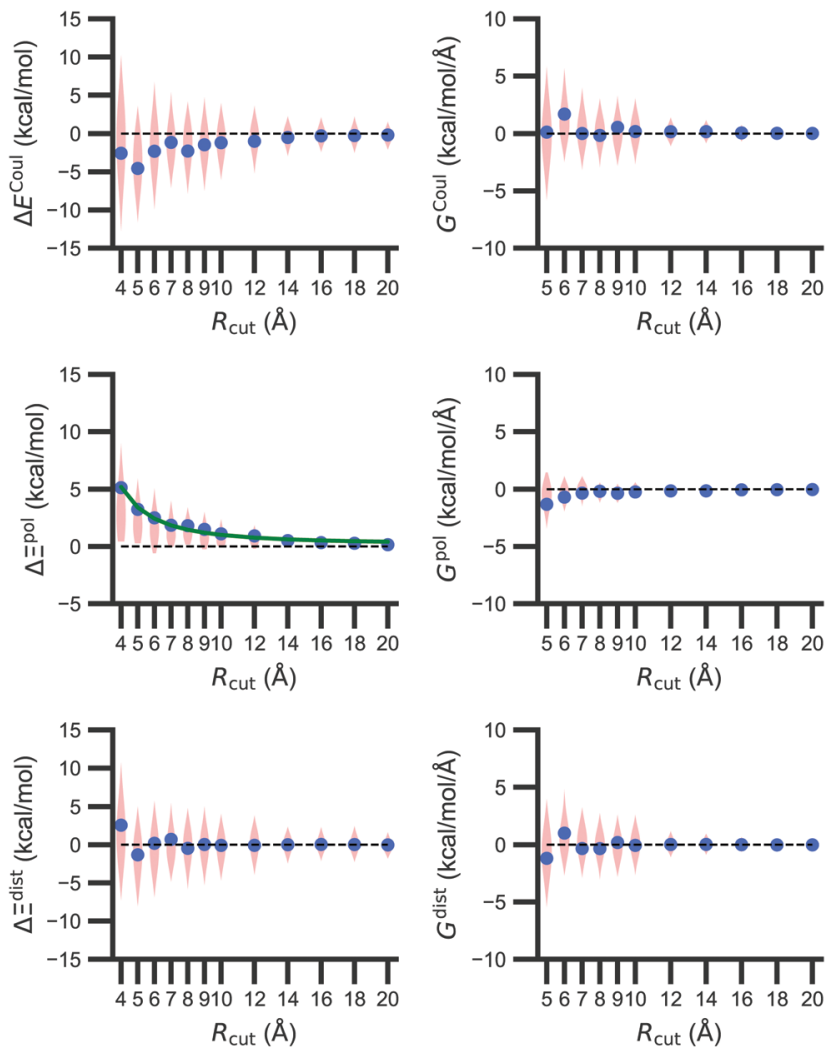

Fig. 5 Dependence of the Coulomb interaction $E^{\text {Coul }}$, the ligand polarization energy $\Xi^{\mathrm{pol}}$, and the distortion energy $\Xi^{\text {dist }}$ on the cutoff distance $R_{\text {cut }}$. Here, the deviation and the gradient are defined as $\Delta F\left(R_{\text {cut }}\right)=F\left(R_{\text {cut }}\right)-F(\infty)$ and $G=\mathrm{d} F\left(R_{\text {cut }}\right) / \mathrm{d} R_{\text {cut }}$, respectively, where $F$ is either $E$ or $\Xi$. In these violin plots, the width of the shaded area is proportional to the frequency of observations. Large blue points are placed at mean values. In the plot of $\Delta \Xi^{\text {pol }}$ as a function of $R_{\text {cut }}$, the green line is a function that was fitted to the mean values, $80.778 R_{\text {cut }}{ }^{-2}+0.177$.

scipy.optimize.curve_fit (https://scipy.org/) of $x_{1} R_{\text {cut }}{ }^{-2}+x_{2}$ for $x_{1}$ and $x_{2}$ yielded a curve that closely matches the data. The curve is best for low $R_{\text {cut }}$, slightly underestimates the mean for intermediate $R_{\text {cut }}$, and slightly overestimates the mean for larger $R_{\text {cut }}$. The inverse square power law is consistent with the $R^{-4}$ dependence of ion-induced dipole interactions because the volume of the region containing embedding field charges increases as $R_{\text {cut }}^{2}$.

3.4 Of computed properties, $\Xi^{\mathrm{pol}}$ is most correlated with the electric field, the induced dipole moment, and the classical polarization energy

We observed that a number of properties - the percentage of atoms in a protein that are highly charged, the number density of highly charged atoms, and the Coulomb interaction energy have little or only weak correlation with the ligand polarization energy (Fig. S3 in the ESI $\dagger$ ). We also observed that the molecular polarizability scalar $\left(\alpha_{\mathrm{L}}\right)$ has a strong linear correlation with the number of electrons in the system (Fig. S4 in the ESI $\dagger$ ) but not with the ligand polarization energy.

In contrast with the aforementioned properties, there is a much clearer relationship between the ligand polarization
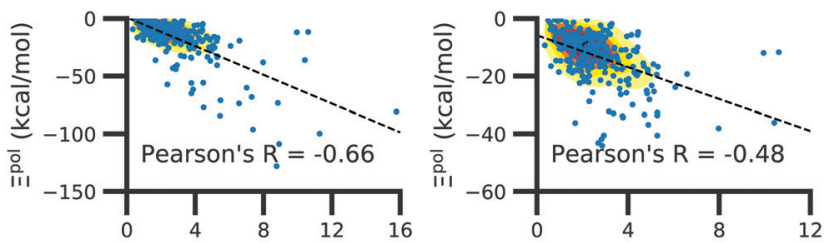

$\left|\mathbf{E}_{L}^{0}\right|(\mathrm{kcal} / \mathrm{mol} / \mathrm{D})$

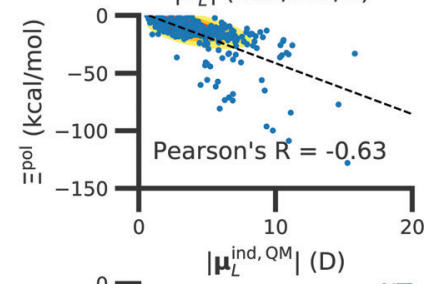

$\left|\mathbf{E}_{L}^{0}\right|(\mathrm{kcal} / \mathrm{mol} / \mathrm{D})$
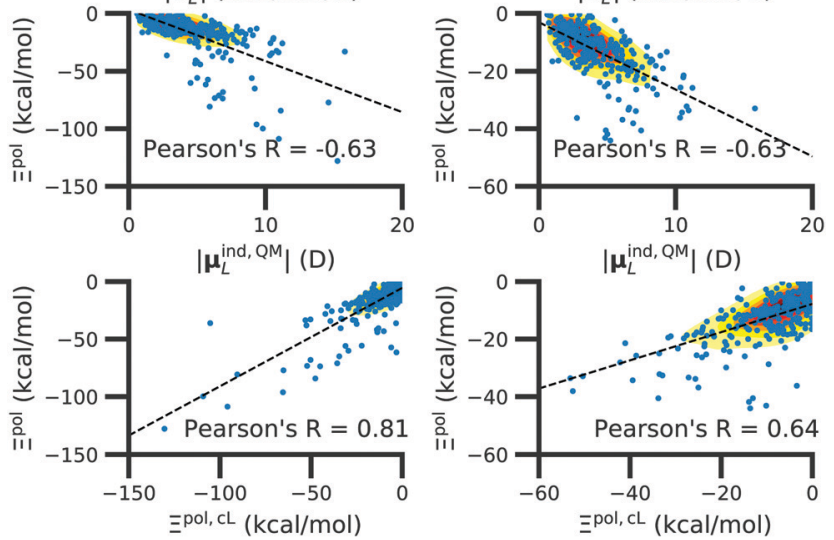

Fig. 6 The ligand polarization energy, $\Xi^{\mathrm{pol}}$, as a function of the magnitude of the electric field $\left|E_{L}^{O}\right|$ (top), the magnitude of the induced dipole moment $\left|\mu_{\mathrm{L}}^{\text {ind, } \mathrm{QM}}\right|$ (middle), and the classical polarization energy $\Xi^{\mathrm{pol}, \mathrm{cL}}$ (bottom), where $\mathbf{E}_{\mathrm{L}}^{O}, \boldsymbol{\mu}_{\llcorner}^{\text {ind, } \mathrm{QM}}$, and $\Xi^{\text {pol,cL }}$ are from eqn (29), (30), and (28), respectively. The range of $\Xi^{\text {pol }}$ is either $\Xi^{\text {pol }}<0 \mathrm{kcal} \mathrm{mol}^{-1}$ (left) or $-50 \mathrm{kcal} \mathrm{mol}^{-1}<$ $\Xi^{\text {pol }}<0 \mathrm{kcal} \mathrm{mol}^{-1}$ (right).

energy, $\Xi^{\mathrm{pol}}$, and several other properties: the magnitude of the electric field; the magnitude of the induced dipole moment of the ligand; and the classical polarization energy (Fig. 6). The linear correlation is strong with the magnitude of the electric field on the ligand center of mass, $\left|\mathbf{E}_{\mathrm{L}}^{0}\right|$, and even stronger with the magnitude of the total electric field vector active on all ligand atoms, $\left|\sum_{A \in L} \mathbf{E}_{A}^{0}\right|$ (Fig. 6 and Fig. S5 in the ESI $\dagger$ ). Intriguingly, in both cases, there appear to be two distinct trends relating the electric field to the magnitude of the electric field; a linear correlation exists in systems where $\Xi^{\mathrm{pol}}<-50 \mathrm{kcal} \mathrm{mol}^{-1}$, but the slope is distinct from in systems where $-50 \mathrm{kcal} \mathrm{mol}^{-1}<$ $\Xi^{\mathrm{pol}}<0 \mathrm{kcal} \mathrm{mol}^{-1}$. The two measures of the electric field are also correlated with each other, with a Pearson's $R$ of 0.54 (Fig. S6 in the ESI $\dagger$ ). Similarly, the ligand polarization energy $\Xi^{\mathrm{pol}}$ is also strongly correlated with the magnitude of the induced dipole moment of the ligand. There is a stronger correlation with the magnitude of the induced dipole moment based on wave functions $\left|\mu_{\mathrm{L}}^{\text {ind,QM }}\right|$, where $\mu_{\mathrm{L}}^{\text {ind,QM }}$ is from eqn (30), than the magnitude of the induced dipole moment based on the molecular polarizability tensor, $\left|\mu_{\mathrm{L}}^{\text {ind, } \alpha_{\mathrm{L}}}\right|$, where $\mu_{\mathrm{L}}^{\text {ind, } \alpha_{\mathrm{L}}}$ is from eqn (31) (Fig. 6 and Fig. S7 in the ESI + ).

Finally, in addition to the strong relationship between the ligand polarization energy $\Xi^{\mathrm{pol}}$ and both the magnitude of the electric field and the induced dipole, there is also a clear correspondence between the ligand polarization energy $\Xi^{\mathrm{pol}}$ and the classical polarization energy. Of approaches to compute the classical polarization energy, treating the entire ligand 
as a dipole and using eqn (30) for the induced dipole moment led to the best correlation with the quantum polarization energy (Fig. 6 and Fig. S8 in the ESI $\dagger$ ). The clear correlation between the two quantities suggests that the classical model of a dipole in an electric field is a reasonable explanation for the quantum behavior. Limitations of the molecular polarizability model are described in Fig. S9 and S10 in the ESI. $\dagger$

The observed linear correlation between the ligand polarization energy and the magnitude of the electric field $\left|\mathbf{E}_{\mathrm{L}}^{0}\right|$ (Fig. 6) has potential implications for modeling protein-ligand interactions with MM, including molecular docking. Because $\left|\mathbf{E}_{\mathrm{L}}^{0}\right|$ is computed without a QM calculation, a relatively inexpensive polarization energy estimate based on linear regression can be added to binding energy estimates. Such an approach could recapitulate some of the success of semi-empirical QM in reconstructing binding poses. ${ }^{12-15}$

\subsection{Polarization is a substantial and variable fraction of interaction and binding energies}

We observe that the ligand polarization energy $\Xi^{\text {pol }}$ can be a substantial and highly system-dependent fraction of the interaction energy and binding energy (Fig. 7). In most systems

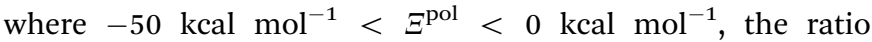
$\Xi^{\mathrm{pol}} / \Xi^{\mathrm{elec}}$ ranges from 0 to 0.4 (Fig. $7 \mathrm{a}$ ). Exceptions occur when $\Xi^{\text {elec }}$ is positive, leading to a negative ratio, or when it is small, leading to a ratio much larger than 1 (Table S2 in the ESI $\dagger$ ). Positive and small values of $\Xi^{\text {elec }}$ result from positive $E^{\text {Coul }}$. For example, the complex $5 \mathrm{c} 2 \mathrm{~h}$ has $\Xi^{\mathrm{pol}}=-22.45 \mathrm{kcal} \mathrm{mol}^{-1}$, $E^{\mathrm{Coul}}=19.60 \mathrm{kcal} \mathrm{mol}^{-1}$, and $\Xi^{\text {elec }}=-2.85 \mathrm{kcal} \mathrm{mol}^{-1}$. Hence, $\Xi^{\mathrm{pol}} / \Xi^{\text {elec }}=-7.88$. The histogram of $\Xi^{\mathrm{pol}} /\left(E^{\mathrm{pair}}+\Xi^{\mathrm{pol}}\right)$ is compressed compared to $\Xi^{\mathrm{pol}} / \Xi^{\text {elec }}$, with the range with the largest density reduced to between 0 and 0.2 (Fig. 7b). Smaller ratios are due to the addition of van der Waals interactions that increase values in the denominator. The histograms of $\Xi^{\mathrm{pol} /}$ $\left(\Psi_{\mathrm{OBC} 2}^{\mathrm{bind}, \mathrm{np}}+\Xi^{\mathrm{pol}}\right)$ and $\Xi^{\mathrm{pol}} / \Psi_{\mathrm{OBC} 2}^{\mathrm{bind}}$ is notable for a clear peak around 0.2 (Fig. $7 \mathrm{c}$ and d). If all systems in the PDBBind are considered, qualitative trends are similar but there is increased density at higher ratios (Fig. S11 in the ESI $\dagger$ ).

When considering the polarization energies of three HIVprotease inhibitors, Hensen et $a l .{ }^{6}$ found that $\Xi^{\mathrm{pol}}$ can approach one-third of the electrostatic interaction energy. In our much larger data set, we found that $\Xi^{\text {pol }}$ can be a larger fraction of $\Xi^{\text {elec }}$.

With the caveat that polarization could be overestimated in these cases, two examples where $\Xi^{\mathrm{pol}} /\left(\Psi_{\mathrm{OBC} 2}^{\mathrm{bind}, \mathrm{np}}+\Xi^{\mathrm{pol}}\right)$ is particularly large, $3 \mathrm{~d} \times 1$ and $3 \mathrm{dx} 2$, highlight the potentially outsized importance of $\Xi^{\mathrm{pol}}$ for small ligands (Table S2 in the ESI $\dagger$ ). In $3 \mathrm{dx} 1, \Psi_{\mathrm{OBC} 2}^{\mathrm{bind}}+\Xi^{\mathrm{pol}}=-12.953 \mathrm{kcal} \mathrm{mol}^{-1}, \Xi^{\mathrm{pol}}=-80.77 \mathrm{kcal}$ $\mathrm{mol}^{-1}$, and the ratio is $\Xi^{\mathrm{pol}} /\left(\Psi_{\mathrm{OBC} 2}^{\mathrm{bind}, \mathrm{np}}+\Xi^{\mathrm{pol}}\right)=6.236$. For comparison, in 2zcq, $\Psi_{\mathrm{OBC} 2}^{\text {bind }}+\Xi^{\text {pol }}=-295.48 \mathrm{kcal} \mathrm{mol}^{-1}$, $\Xi^{\mathrm{pol}}=-128.01 \mathrm{kcal} \mathrm{mol}^{-1}$, and the ratio is $\Xi^{\mathrm{pol}} /\left(\Psi_{\mathrm{OBC} 2}^{\mathrm{bind}, \mathrm{np}}+\Xi^{\mathrm{pol}}\right)=$ 0.43 . The ligand in $3 \mathrm{~d} \times 1$ (Fig. 4 ) is much smaller than the ligand in 2zcq (Fig. 8). Small ligands have fewer opportunities for pairwise contacts with their protein binding partners than larger ligands. The limited number of contacts leads to a weaker $\Psi_{\mathrm{OBC} 2}^{\text {bind,np }}$, such that $\Xi^{\mathrm{pol}}$ can play a larger role.
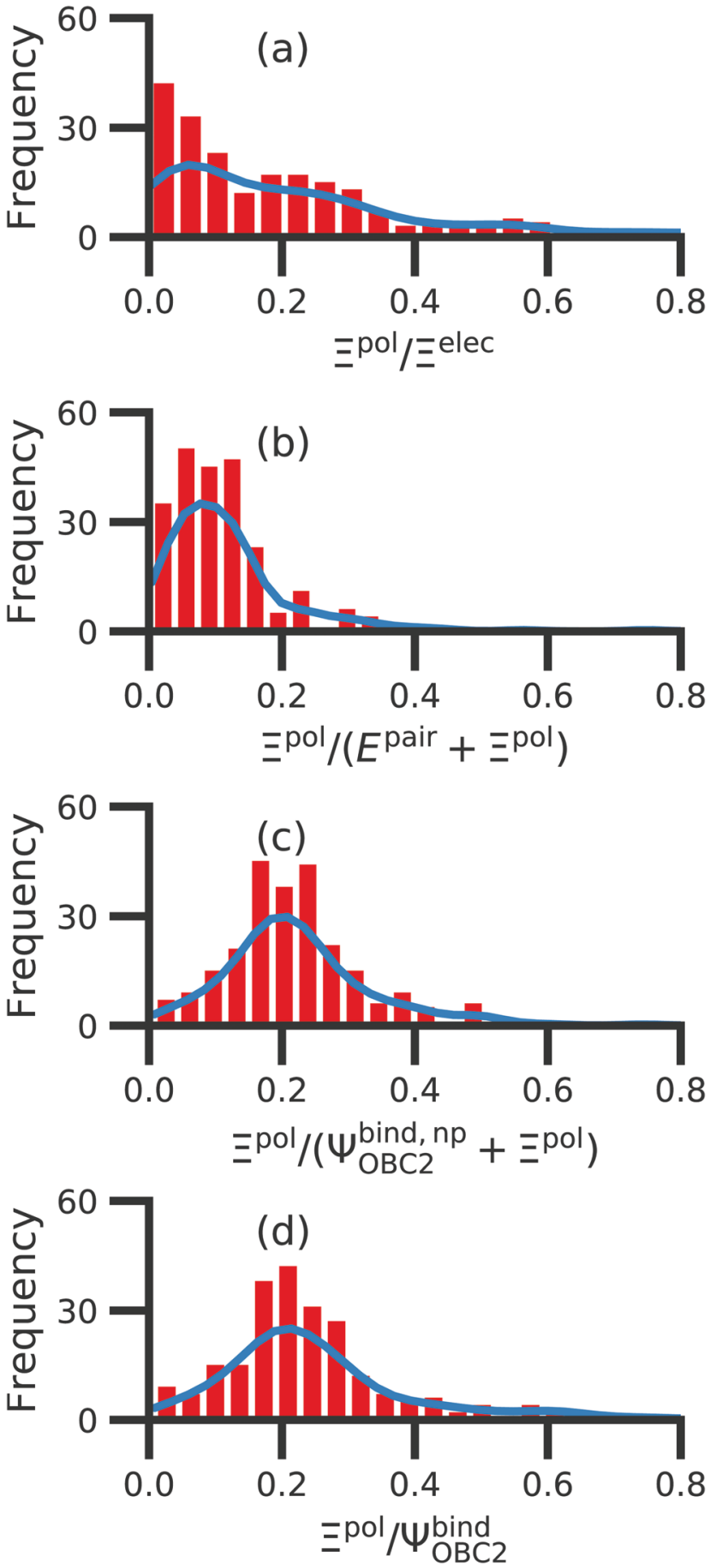

Fig. 7 Histograms of ratio of the polarization energy of the ligand to (a) the electrostatic interaction $\left(\Xi^{\text {elec }}=E^{\text {Coul }}+\Xi^{\text {pol }}\right.$ ), (b) the intermolecular pairwise potential energy with the ligand polarization energy $\left(E^{\text {pair }}+\Xi^{\text {pol }}\right)$, (c) the binding energy without considering ligand polarization in the solvation free energy $\left(\Psi_{\mathrm{OBC2}}^{\text {bind, }} \mathrm{p}+\Xi^{\mathrm{pol}}\right)$, and $(\mathrm{d})$ the binding energy with considering ligand polarization in the solvation free energy $\left(\Psi_{\mathrm{OBC2}}^{\text {bind }}\right)$. The histograms are truncated at a ratio of 1.25. Data are only included for complexes where $\Xi^{\text {pol }}<0 \mathrm{kcal} \mathrm{mol}^{-1}$ (left) or $-50 \mathrm{kcal} \mathrm{mol}^{-1}<\Xi^{\text {pol }}<0 \mathrm{kcal} \mathrm{mol}^{-1}$. For analogous histograms including all data, see Fig. S11 in the ESI. $\dagger$

The relative importance of ligand polarization in small ligands may explain the poor performance of binding free 


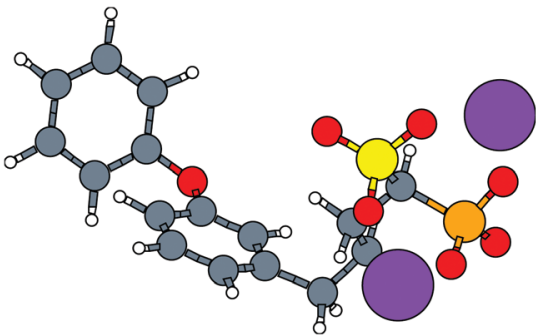

Fig. 8 The molecular structure of the ligand with two magnesium cations $\mathrm{Mg}^{2+}$ in the complex, 2zcq. Hydrogen, carbon, oxygen, magnesium, phosphorus, and sulfur atoms are colored with white, gray, red, pink, orange, and yellow, respectively.

energy methods based on a fixed-charge force field in distinguishing molecules that are active and inactive against T4 lysozyme L99A. ${ }^{46}$ In this protein, the L99A mutation forms a pocket known to bind a number of small hydrophobic compounds. Xie et al. $^{46}$ performed binding free energy calculations for a library of 141 small hydrophobic compounds whose thermal activity against T4 lysozyme L99A had been measured. Many of the compounds contained highly polarizable aromatic groups. The best-performing method in Xie et al. ${ }^{46}$ had an area under the receiver operating characteristic curve of 0.74 (0.04) out of 1 for a perfect binary classifier. Binary classification performance could potentially be improved by incorporating the ligand polarization, as described in the current paper.

\subsection{Solvent usually has a small effect on ligand polarization}

In the vast majority of systems, polarization of the ligand is similar whether solvent is considered or not (Fig. 9). The difference between $\Xi_{\mathrm{sol}}^{\mathrm{pol}}$ and $\Xi^{\mathrm{pol}}$ has a mean of $2.0 \mathrm{kcal} \mathrm{mol}^{-1}$ and standard deviation of $6 \mathrm{kcal} \mathrm{mol}^{-1}$. Only a small percentage $(7 \%)$ of the scatter plot for the two quantities deviate from linearity $(y=x)$ by more than $10 \mathrm{kcal} \mathrm{mol}^{-1}$, such that $\mid \Xi_{\text {sol }}^{\text {pol }}-$ $\Xi^{\mathrm{pol}} \mid>10 \mathrm{kcal} \mathrm{mol}^{-1}$ (Fig. S12 in the ESI $\dagger$ ). While the scatter

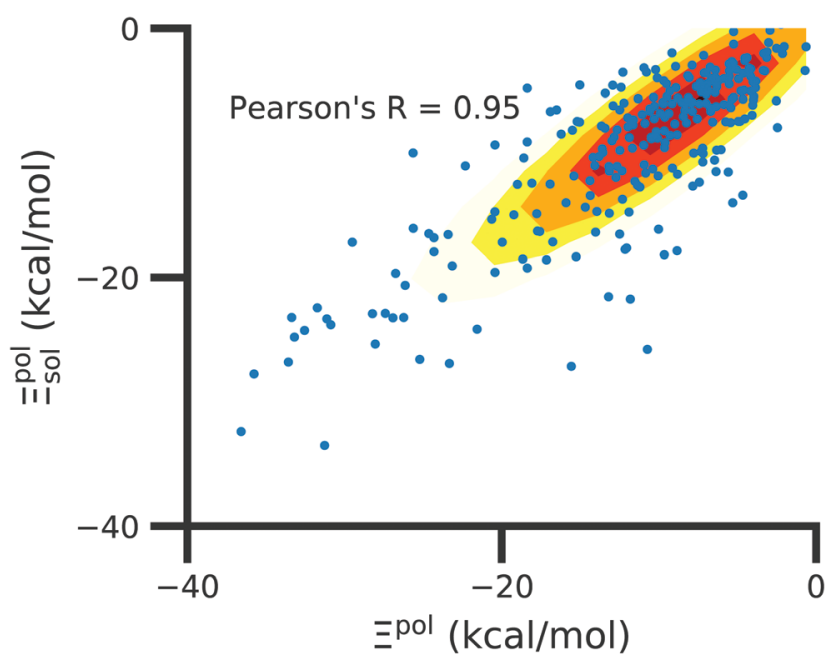

Fig. 9 Solvent effect on the ligand polarization energies of the ligandprotein complexes. The axes are limited to a range of $-40 \mathrm{kcal} \mathrm{mol}^{-1}<$ $\Xi^{\text {pol }}<0$ kcal mol . $^{-1}$. plot focuses on the range where $-40 \mathrm{kcal} \mathrm{mol}^{-1}<\Xi_{\text {sol }}^{\text {pol }}<$ $0 \mathrm{kcal} \mathrm{mol}{ }^{-1}$, the same trend, in which the embedding field dominates ligand polarization energies, holds true for even lower $\Xi_{\text {sol }}^{\text {pol }}$ In most complexes, ligands are embedded in their respective receptors. When the ASCs are far from the ligand, they have a minimal effect on the electric field experienced by the ligand and on the ligand polarization energy.

There are small number of systems in which the ligand is polarized by the solvent much more so than by the protein. These are exceptions rather than the rule. They can only occur when the ligand is exposed to the surface and the protein generates a strong electrostatic potential at the surface.

Further underscoring the relatively limited effect of solvent on ligand polarization energies, solvation free energies calculated with and without polarization by solvent are highly correlated (Fig. 10). If solvent had a large effect on ligand polarization, the solvation free energy estimate from the OBC2 model, which does not treat solvent-induced ligand polarization, would have a markedly different trend than ddCOSMO model, which does. Instead, the Pearson R between the two estimates is nearly 1.

Although the solvation free energy estimates are very correlated, there is small systematic difference between the implicit solvent models. For the ligand, protein, complex, and binding energy, the slope (and intercept) are 0.94 (5.7), 1.04 (121.6), 1.04 (99.2), and $0.96(-18.1)$. The shift in the intercept suggests a systematic error in one of the models.

\subsection{Solvation and polarization can be key drivers of native complex formation}

For a number of native complexes, both polarization and solvation were required to compute negative binding energies (Fig. 11).
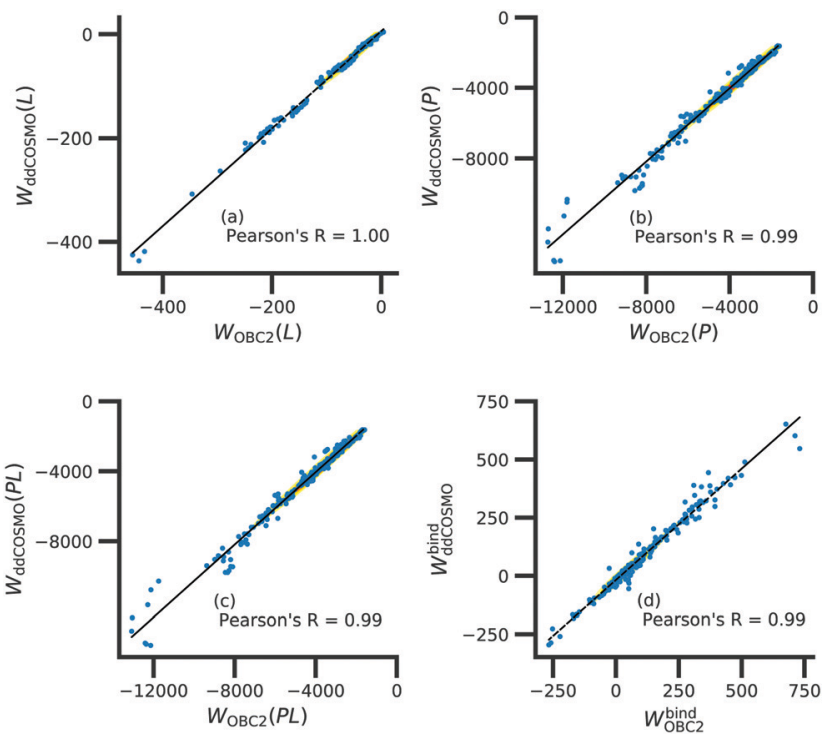

Fig. 10 Comparison of solvation free energy estimates (in $\mathrm{kcal} \mathrm{mol}^{-1}$ ) based on OBC2 ( $x$-axis) and ddCOSMO ( $y$-axis). Solvation free energy estimates are of the (a) ligand, (b) protein, (c) complex, and (d) the binding energy. 

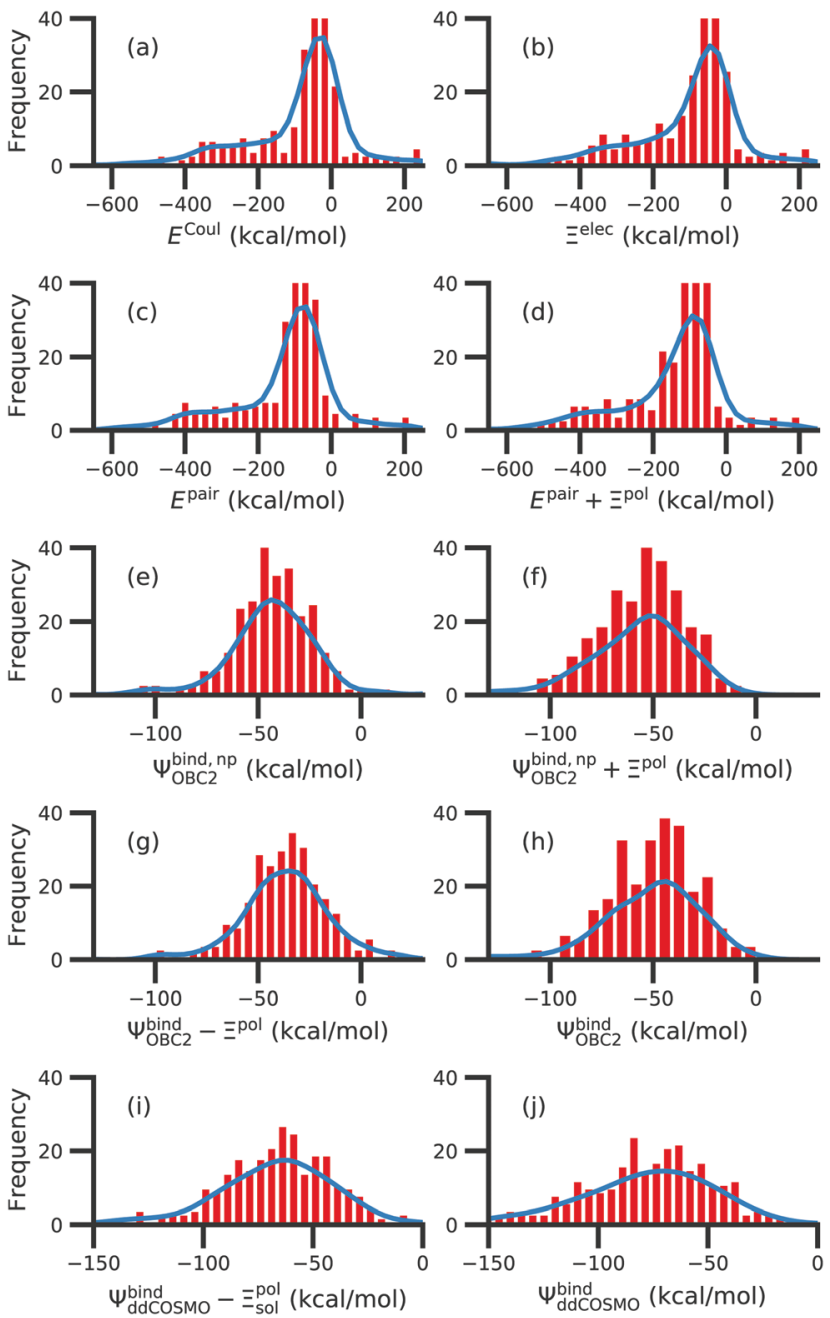

Fig. 11 Histograms of intermolecular potential energies and binding energies. The intermolecular potential energies are (a) the permanent Coulomb interaction ( $E^{\text {Coul }}$ ), (b) the electrostatic interaction $\left(\Xi^{\text {elec }}=\right.$ $\left.E^{\text {Coul }}+\Xi^{\text {pol }}\right)$, (c) the intermolecular pairwise potential energy $\left(E^{\text {pair }}=\right.$ $E^{\mathrm{vdW}}+E^{\text {Coul }}$, and (d) the intermolecular pairwise potential energy with the polarization energy of the ligand $\left(E^{\text {pair }}+\Xi^{\text {pol }}\right)$ in the gas phase. The OBC2 binding energies are (e) without considering ligand polarization at all, $\Psi_{\mathrm{OBC} 2}^{\text {bind }}$, (f) considering ligand polarization for electrostatic interactions but not in the solvation free energy, $\Psi_{\mathrm{OBC} 2}^{\text {bind, np }}+\Xi^{\mathrm{pol}},(\mathrm{g})$ considering ligand polarization in the solvation free energy but not for electrostatic interactions, $\Psi_{\mathrm{OBC} 2}^{\text {bind }}-\Xi^{\mathrm{pol}}$, (h) considering ligand polarization both in the electrostatic interactions and the solvation free energy. The ddCOSMO binding energies are (i) without and (j) with the ligand polarization energy. A similar plot that only considers systems for which $-50<\Xi^{\text {pol }}<$ $0 \mathrm{kcal} \mathrm{mol}{ }^{-1}$ is available as Fig. S13 in the ESI. $\dagger$

Due to the harmonic restraint maintained during minimization, our models closely resemble their native crystal structures. In order for these protein-ligand complexes to adopt these structures, they should have a negative binding energy (presuming that binding results in entropy loss). Intriguingly, the Coulomb interaction energy is positive in a significant fraction of these systems (Fig. 11a). Incorporating van der Waals interactions in $E^{\text {pair }}$ slightly reduces the number of systems in which the interaction energy is positive (Fig. 11c). However, these pairwise terms, which are standard to molecular docking, are insufficient to accurately describe all the native complexes with a negative interaction energy. Incorporating a ligand polarization term (Fig. 11b and d) or nonpolarizable solvation energy term (Fig. 11e and g) alone is also insufficient. However, when both polarization and solvation are considered, all the native complexes have a negative binding energy (Fig. 11f, h, i and j) (Fig. 11i removes ligand polarization but the solvation energy still considers polarization.) Considering both polarization and solvation terms also appears to attenuate the broad range of binding energies observed in $E^{\text {pair }}, E^{\text {pair }}+\Xi^{\text {pol }}$, and $\Psi^{\text {bind,np }}$ (Fig. 11f, h, i and j). Using the partial charges $q_{A}^{\text {QM }}$ opposed to $q_{A}^{\mathrm{QM}: Q_{I}}$ does not have a qualitative effect on these trends. The trends also hold for systems within the normal range of $-50<$ $\Xi^{\mathrm{pol}}<0 \mathrm{kcal} \mathrm{\textrm {mol } ^ { - 1 }}$ (Fig. S13 in the ESI $\dagger$ ). The importance of including ligand polarization and solvation was previously noted by Kim and Cho, ${ }^{17}$ who achieved superior performance at binding pose prediction using a protocol that combined atomic charges from $\mathrm{QM} / \mathrm{MM}$ with solvation compared to using either by themselves.

The lowest $E^{\text {Coul }}$ are due to phosphate groups. The lowest $E^{\text {Coul }}$ is observed in the complex 2zcq. The complex contains two $\mathrm{Mg}^{2+}$ in close proximity to a negatively-charged phosphate group (Fig. 8). The complex $1 \mathrm{u} 1 \mathrm{~b}$ also has a very low $E^{\text {Coul }}$. The ligand in $1 \mathrm{u} 1 \mathrm{~b}$ contains four phosphates (Fig. S14 in the ESI $\dagger$ ). RESP charges on the phosphorus are around 1.4e and oxygen charges range from -0.4 to $0.8 e$, leading to a low $E^{\mathrm{Coul}}$.

\subsection{Solvation but not polarization improves correlation with experimental binding free energies}

An important goal in protein-ligand modeling is the accurate calculation of binding free energies - which quantify the strength of noncovalent association - that are consistent with experimentally observed values.

For several reasons, the computed binding energy $\Delta G^{\text {bind }}$ is not expected to completely agree with the experimentally measured binding free energy $\Delta G^{\text {bind }}$ for complexes in the PDBBind Core Set. These reasons include that:

- The binding free energy $\Delta G^{\text {bind }}$ is not rigorously equivalent to $\Psi^{\text {bind }}$, but is actually an exponential average over the ensemble of the complex. ${ }^{47,48}$ Using $\Psi^{\text {bind }}$ to model $\Delta G^{\text {bind }}$ is an approximation that neglects entropy.

- The binding energy model is not exact. For example, the present model does not explicitly treat polarization of the free ligand by solvent, polarization of the protein by the ligand, and the solvation model does not include explicit water.

- The PDBBind is a heterogeneous data set in which experimental $\Delta G^{\text {bind }}$ were determined by various modalities and under different experimental conditions. There may be systematic differences between measured $\Delta G^{\text {bind }}$ that are not considered in our models.

- On a related note, experimental conditions used to obtain crystal structures and binding affinity data are different. Crystal structures have packing forces and are generally at a lower temperature. 
Nonetheless, a comparison between computed interaction energies and experimental binding free energies can be informative.

While the treatment of solvation is essential, ligand polarization energies have a minimal effect on the correlation between $\Psi^{\text {bind }}$ and experimental $\Delta G^{\text {bind }}$ (Fig. 12 and Fig. S15 in the ESI $\dagger$ ). If solvation energies are not considered, the distribution of intermolecular pairwise potential energies $E^{\text {pair }}$ of the protein-ligand complexes is distributed extremely broadly from $-1000 \mathrm{kcal} \mathrm{mol}^{-1}$ to $250 \mathrm{kcal} \mathrm{mol}^{-1}$ and the
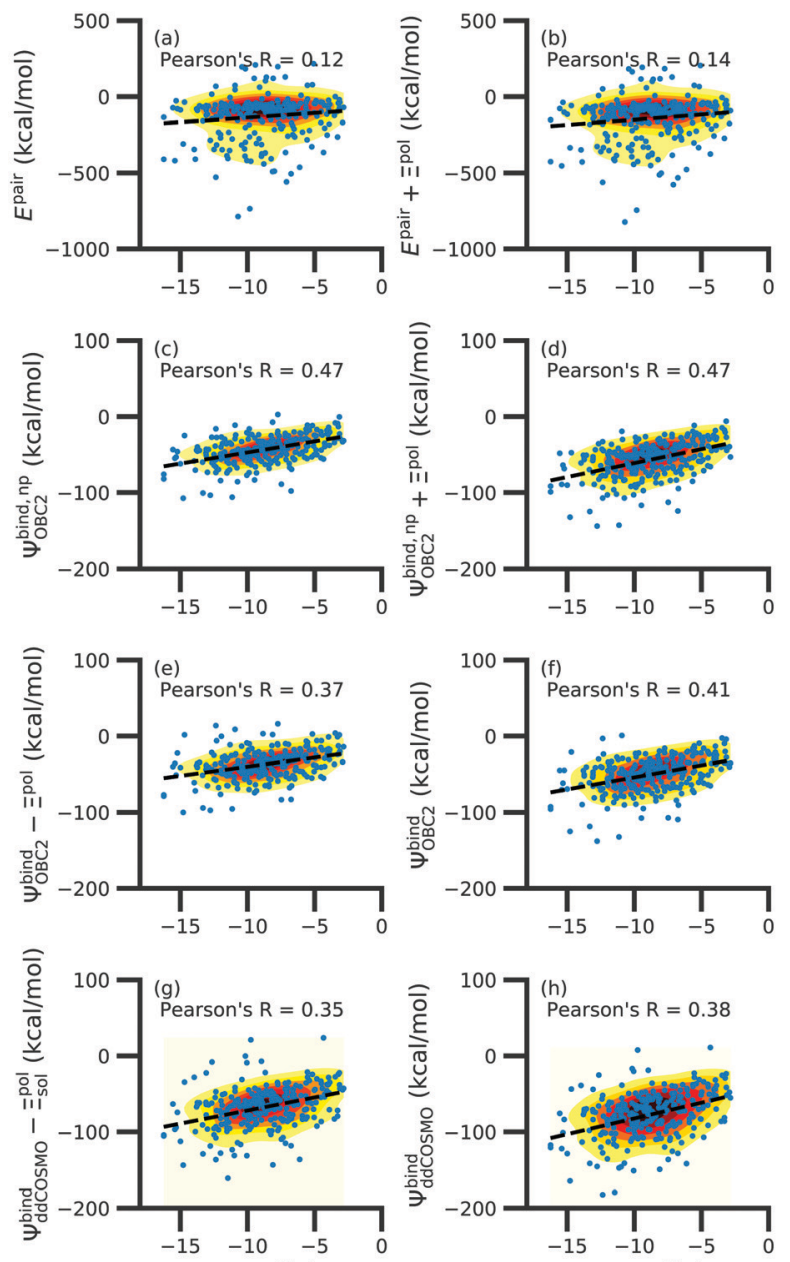

Experimental $\Delta G^{\text {bind }}(\mathrm{kcal} / \mathrm{mol}) \quad$ Experimental $\Delta G^{\text {bind }}(\mathrm{kcal} / \mathrm{mol})$

Fig. 12 Comparison of interaction energies (in $\mathrm{kcal} \mathrm{mol}^{-1}$ ) to experimentally measured binding free energies (in $\mathrm{kcal} \mathrm{mol}^{-1}$ ) for complexes with $-50 \mathrm{kcal} \mathrm{mol}^{-1}<\Xi^{\mathrm{pol}}<0 \mathrm{kcal} \mathrm{mol}^{-1}$. Interaction energies are according to (a) the intermolecular pairwise potential energy ( $\left.E^{\text {pair }}=E^{\mathrm{vdW}}+E^{\text {Coul }}\right)$ and (b) the intermolecular pairwise potential energy with the polarization energy of the ligand ( $\left.E^{\text {pair }}+\Xi^{\text {pol }}\right)$ in the gas phase. Panels $(c-h)$ are binding energies, with $(\mathrm{c}-\mathrm{f})$ based on the OBC2 and ( $\mathrm{g}$ and $\mathrm{h}$ ) based on the ddCOSMO implicit solvent models. The OBC2-based binding energies are: (c) Without considering ligand polarization at all, $\Psi_{\mathrm{OBC} C}^{\text {bind }}$; (d) considering ligand polarization for electrostatic interactions but not in the solvation free energy, $\Psi^{\text {bind,np }}+\Xi^{\text {pol. }}$; (e) considering ligand polarization in the solvation free energy but not for electrostatic interactions, $\Psi^{\text {bind }}-\Xi^{\text {pol}}$; or ( $f$ ) considering ligand polarization both in the electrostatic interactions and the solvation free energy. The ddCOSMO-based binding energies are (g) without and (h) with considering the ligand polarization energy. A similar plot for all complexes is available as Fig. S15 in the ESI. $\dagger$ correlation between $\Psi^{\text {bind }}$ and experimental $\Delta G^{\text {bind }}$ is negligible (Fig. 12a, b and Fig. S15a, b in the ESI $\dagger$ ). Incorporating solvation but not polarization significantly improves Pearson's $R$ to 0.47 for complexes where $-50 \mathrm{kcal} \mathrm{mol}^{-1}<\Xi^{\text {pol }}<$ $0 \mathrm{kcal} \mathrm{mol}{ }^{-1}$ and 0.44 for complexes where $\Xi^{\mathrm{pol}}<0 \mathrm{kcal} \mathrm{mol}^{-1}$ (Fig. 12c and Fig. S15c in the ESI $\dagger$ ). Although the range of computed binding energies is dramatically reduced to

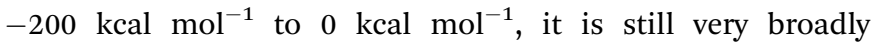
distributed compared to the distribution of experimentally measured binding free energies $\left(-16 \mathrm{kcal} \mathrm{mol}^{-1}<\Delta G^{\text {bind }}<\right.$ $-3 \mathrm{kcal} \mathrm{mol}^{-1}$ ), supporting the idea that a single structure cannot represent an ensemble of structures obtained in experimental conditions. Adding the polarization energy to solvation energies computed without solvation has no effect on the solvation energy (Fig. 12d and Fig. S15d in the ESI $\dagger$ ). In comparison, computing solvation energies using partial charges from the induced dipole diminishes correlation with experiment (Fig. 12e, f and Fig. S15e, f in the ESI $\dagger$ ). The solvation energy computed with ddCOSMO demonstrates similar performance (Fig. 12g, h and Fig. S15g, h in the ESI $\dagger$ ).

In a critical assessment of a number of docking programs and scoring functions across eight different diverse proteins, Warren et $a l .{ }^{49}$ concluded that "no statistically significant relationship existed between docking scores and ligand affinity." Our data suggest that the lack of correlation stems from a poor or nonexistent treatment of solvation in the scoring functions. Perhaps due to cancellation of error, neglect of ligand polarization does not appear to be a major factor in the poor performance of docking scores.

\section{Conclusions}

Using $\mathrm{QM} / \mathrm{MM}^{6,23,50,51}$ with and without ddCOSMO implicit solvent, ${ }^{30,31}$ we computed polarization energies $\left(\Xi^{\mathrm{pol}}\right.$ and $\left.\Xi_{\mathrm{sol}}^{\mathrm{pol}}\right)$ for 286 complexes in the PDBBind Core Set. ${ }^{25}$ The distributions of $\Xi^{\text {pol }}, \Xi^{\text {dist }}$, and $\Xi^{\text {stab }}$ were found to be broad and skewed. For properly prepared systems without atoms in unrealistically close contact, these terms all have the expected sign of $\Xi^{\text {pol }}<0, \Xi^{\text {dist }}>0$, and $\Xi^{\text {stab }}<0$. The lowest $\Xi^{\text {pol }}$ were observed in systems where cations are close to ligand atoms. In these systems, the extent of polarization is likely to be overestimated. The importance of including embedding field charges on $\Xi^{\mathrm{pol}}$ appears to diminish, on average, as an inverse square law. There is no clear relationship between $\Xi^{\text {pol }}$ and the percentage of highly charged atoms in a protein and molecular polarizability scalar. There is a weak correlation between $\Xi^{\text {pol }}$ and the Coulomb energy $E^{\text {Coul }}$. On the other hand, there is a stronger linear correlation between $\Xi^{\mathrm{pol}}$ and the magnitude of the electric field, the magnitude of the induced dipole moment, and the classical polarization energy. The ligand polarization energy $\Xi^{\mathrm{pol}}$ is observed to a substantial and system-dependent fraction of the electronic interaction energy and the total interaction energy. In most cases, the effect of the implicit solvent on the ligand polarization energy is minor. In some systems, consideration of ligand polarization and solvation are 
both essential for calculating negative interaction energies for crystallographic complexes. While consideration of solvation is essential for achieving moderate correlation between interaction energies and experiment, we did not observe that the ligand polarization energy $\Xi^{\text {pol }}$ improves the correlation between the binding energy and experimental binding free energies.

\section{Conflicts of interest}

There are no conflicts to declare.

\section{Acknowledgements}

We thank Pengyu Ren for the suggestion to compare polarization energies with molecular polarizability. We thank Filippo Lipparini and Qiming Sun for valuable discussions and implementation of QM/MM/ddCOSMO. We thank OpenEye Scientific Software, Inc. for providing academic licenses to their software. This research was supported by the National Institutes of Health (R01GM127712).

\section{Notes and references}

1 D. Jimenez-Morales, J. Liang and B. Eisenberg, Eur. Biophys. J., 2012, 41, 449-460.

2 W. M. Haynes, CRC Handbook of Chemistry and Physics, CRC Press, 94th edn, 2016.

3 B. Honig and A. Nicholls, Science, 1995, 268, 1144-1149.

4 M. Garcia-Viloca, D. G. Truhlar and J. Gao, J. Mol. Biol., 2003, 327, 549-560.

5 M. W. van der Kamp, F. Perruccio and A. J. Mulholland, Proteins: Struct., Funct., Bioinf., 2007, 69, 521-535.

6 C. Hensen, J. C. Hermann, K. Nam, S. Ma, J. Gao and H.-D. Höltje, J. Med. Chem., 2004, 47, 6673-6680.

7 Y. Shi, Z. Xia, J. Zhang, R. Best, C. Wu, J. W. Ponder and P. Ren, J. Chem. Theory Comput., 2013, 9, 4046-4063.

8 Z. Jing, C. Liu, S. Y. Cheng, R. Qi, B. D. Walker, J.-P. Piquemal and P. Ren, Annu. Rev. Biophys., 2019, 48, 371-394.

9 D. Jiao, P. A. Golubkov, T. A. Darden and P. Ren, Proc. Natl. Acad. Sci. U. S. A., 2008, 105, 6290-6295.

10 U. Ryde and P. Söderhjelm, Chem. Rev., 2016, 116, 5520-5566.

11 A. Crespo, A. Rodriguez-Granillo and V. T. Lim, Curr. Top. Med. Chem., 2017, 17, 2663-2680.

12 P. Chaskar, V. Zoete and U. F. Röhrig, J. Chem. Inf. Model., 2014, 54, 3137-3152.

13 A. Pecina, R. Meier, J. Fanfrlík, M. Lepšík, J. Řezáč, P. Hobza and C. Baldauf, Chem. Commun., 2016, 52, 3312-3315.

14 A. Pecina, S. Haldar, J. Fanfrlík, R. Meier, J. Řezáč, M. Lepšík and P. Hobza, J. Chem. Inf. Model., 2017, 57, 127-132.

15 H. Ajani, A. Pecina, S. M. Eyrilmez, J. Fanfrlík, S. Haldar, J. Řezáč, P. Hobza and M. Lepšík, ACS Omega, 2017, 2, 4022-4029.
16 A. E. Cho, V. Guallar, B. J. Berne and R. Friesner, J. Comput. Chem., 2005, 26, 915-931.

17 M. Kim and A. E. Cho, Phys. Chem. Chem. Phys., 2016, 18, 28281-28289.

18 S. Y. Willow, X. C. Zeng, S. S. Xantheas, K. S. Kim and S. Hirata, J. Phys. Chem. Lett., 2016, 7, 680-684.

19 S. Yoo, Y. A. Lei and X. C. Zeng, J. Chem. Phys., 2003, 119, 6083-6091.

20 T.-M. Chang and L. X. Dang, Chem. Rev., 2006, 106, 1305-1322.

21 C. Caleman, J. S. Hub, P. J. van Maaren and D. van der Spoel, Proc. Natl. Acad. Sci. U. S. A., 2011, 108, 6838-6842.

22 P. Bajaj, A. W. Götz and F. Paesani, J. Chem. Theory Comput., 2016, 12, 2698-2705.

23 J. Gao and X. Xia, Science, 1992, 258, 631-635.

24 P. Fong, J. P. McNamara, I. H. Hillier and R. A. Bryce, J. Chem. Inf. Model., 2009, 49, 913-924.

25 Z. Liu, M. Su, L. Han, J. Liu, Q. Yang, Y. Li and R. Wang, Acc. Chem. Res., 2017, 50, 302-309.

26 A. Szabo and N. S. Ostlund, Modern Quantum Chemistry, Dover Publications, 1996.

27 R. Krishnan, J. Binkley, R. Seeger and J. Pople, J. Chem. Phys., 1980, 72, 650-654.

28 C. I. Bayly, P. Cieplak, W. Cornell and P. A. Kollman, J. Phys. Chem., 1993, 97, 10269-10280.

29 A. Onufriev, D. Bashford and D. A. Case, Proteins: Struct., Funct., Bioinf., 2004, 55, 383-394.

30 F. Lipparini, B. Stamm, E. Cancés, Y. Maday and B. Mennucci, J. Chem. Theory Comput., 2013, 9, 3637-3648.

31 F. Lipparini, G. Scalmani, L. Lagardére, B. Stamm, E. Cancés, Y. Maday, J.-P. Piquemal, M. J. Frish and B. Mennucci, J. Chem. Phys., 2014, 141, 184108.

32 M. L. Connolly, Science, 1983, 221, 709-713.

33 T. J. Richmond, J. Mol. Biol., 1984, 178, 63-89.

34 A. Bondi, J. Phys. Chem., 1964, 68, 441-451.

35 A. Klamt, Wiley Interdiscip. Rev.: Comput. Mol. Sci., 2018, 8, e1338.

36 M. Schaefer, C. Bartels, F. Leclerc and M. Karplus, J. Comput. Chem., 2001, 22, 1857-1879.

37 S. Y. Willow, M. A. Salim, K. S. Kim and S. Hirata, Sci. Rep., 2015, 5, 14358.

38 D. Case, D. Cerutti, I. T. E. Cheatham, T. Darden, R. Duke, T. Giese, H. Gohlke, A. Goetz, D. Greene, N. Homeyer, S. Izadi, A. Kovalenko, T. Lee, S. LeGrand, P. Li, C. Lin, J. Liu, T. Luchko, R. Luo, D. Mermelstein, K. Merz, G. Monard, H. Nguyen, I. Omelyan, A. Onufriev, F. Pan, R. Qi, D. Roe, A. Roitberg, C. Sagui, C. Simmerling, W. Botello-Smith, J. Swails, R. Walker, J. Wang, R. Wolf, X. Wu, L. Xiao, D. York and P. Kollman, AMBER 2017, University of California, San Francisco, 2017.

39 J. A. Maier, C. Martinez, K. Kasavajhala, L. Wickstrom, K. E. Hauser and C. Simmerling, J. Chem. Theory Comput., 2015, 11, 3696-3713.

40 J. Wang, W. Wang, P. A. Kollman and D. A. Case, J. Mol. Graphics Modell., 2006, 25, 247-260.

41 P. Eastman and V. S. Pande, Comput. Sci. Eng., 2010, 12, 34-39. 
42 E. F. Valeev, Libint: A Library for the Evaluation of Molecular Integrals of Many-Body Operators over Gaussian Functions, 2017, version 2.4.2.

43 C. Sanderson and R. Curtin, J. Open Source Software, 2016, $1,26$.

44 C. Sanderson and R. Curtin, in Mathematical Software ICMS 2018, ed. J. H. Davenport, M. Kauers, G. Labahn and J. Urban, Springer International Publishing, Cham, 2018, vol. 10931, pp. 422-430.

45 Q. Sun, T. C. Berkelbach, N. S. Blunt, G. H. Booth, S. Guo, Z. Li, J. Liu, J. McClain, E. R. Sayfutyarova, S. Sharma, S. Wouters and G. K.-L. Chan, Wiley Interdiscip. Rev.: Comput. Mol. Sci., 2018, 8, e1340.
46 B. Xie, T. H. Nguyen and D. D. L. Minh, J. Chem. Theory Comput., 2017, 13, 2930-2944.

47 E. Gallicchio, M. Lapelosa and R. M. Levy, J. Chem. Theory Comput., 2010, 6, 2961-2977.

48 W. Menzer, C. Li, W. Sun, B. Xie and D. D. L. Minh, J. Chem. Theory Comput., 2018, 14, 6035-6049.

49 G. L. Warren, C. V. W. Andrews, A.-M. Capelli, B. Clarke, J. LaLonde, M. H. Lambert, M. Lindvall, N. Nevins, S. F. Semus, S. Senger, G. Tedesco, I. D. Wall, J. M. Woolven, C. E. Peishoff and M. S. Head, J. Med. Chem., 2006, 49, 5912-5931.

50 M. J. Field, P. A. Bash and M. Karplus, J. Comput. Chem., 1990, 11, 700-733.

51 J. Gao, Acc. Chem. Res., 1996, 29, 298-305. 